\title{
A Sediment Graph Model Based on SCS-CN Method
}

\author{
P K Bhunya ${ }^{1}$, Ronny Berndtsson², \\ Raj Deva Singh ${ }^{1}$ and S.N.Panda ${ }^{3}$ \\ ${ }^{1}$ National Institute of Hydrology, Roorkee, Uttarakhand \\ ${ }^{2}$ Dept. of Water Resources Engineering, Lund University, Lund, \\ Indian Institute of Technology, Kharagpur WB \\ 1,3India \\ 2Sweden
}

\section{Introduction}

Sediment is fragmented material primarily formed by the physical and chemical disintegration of rocks from the earth's crust. For example, physical disintegration means where the material is broken down by human interference or can be due to the construction or engineering works. Similarly chemical disintegration is by chemicals in fluids, wind, water or ice and/or by the force of gravity acting on the particle itself. The estimation of sediment yield is needed for studies of reservoir sedimentation, river morphology and soil and water conservation planning. However, sediment yield estimate of a watershed is difficult as it results due to a complex interaction between topographical, geological and soil characteristics. In spite of extensive studies on the erosion process and sediment transport modelling, there exists a lack of universally accepted sediment yield formulae (Bhunya et al. 2010). The conditions that will transport sediment are needed for engineering problems, for example, during canal construction, channel maintenance etc. Interpreting ancient sediments; most sediments are laid down under processes associated with flowing water like rivers, ocean currents and tides.

Usually, the transport of particles by rolling, sliding and saltating is called bed-load transport, while the suspended particles are transported as suspended load transport. The suspended load may also include the fine silt particles brought into suspension from the catchment area rather than from, the streambed material (bed material load) and is called the wash load. An important characteristic of wash load is that its concentration is approximately uniform for all points of the cross-section of a river. This implies that only a single point measurement is sufficient to determine the cross-section integrated wash-load transport by multiplying with discharge. In estuaries clay and silt concentrations are generally not uniformly distributed.

Bed load refers to the sediment which is in almost continuous contact with the bed, carried forward by rolling, sliding or hopping. Suspended load refers to that part of the total sediment transport which is maintained in suspension by turbulence in the flowing water for considerable periods of time without contact with the stream bed. It moves with practically 
the same velocity as that of the flowing water. That part of the suspended load which is composed of particle sizes smaller than those found in appreciable quantities in the bed material. It is in near-permanent suspension and therefore, is transported through the stream without deposition. The discharge of the wash load through a reach depends only on the rate with which these particles become available in the catchment area and not on the transport capacity of the flow. Fluid flow and sediment transport are obviously linked to the formation of primary sedimentary structures. Here in this chapter, we tackle the question of how sediment moves in response to flowing water that flows in one direction.

\section{Fluid flow and sediment transport}

The action of sediment transport which is maintained in the flowing water is typically due to a combination of the force of gravity acting on the sediment and/or the movement of the fluid. A schematic diagram of these forces in a flowing water is shown in Figure 1. The bottom plate is fixed and the top plate is accelerated by applying some force that acts from left to right. The upper plate will be accelerated to some terminal velocity and the fluid between the plate will be set into motion. Terminal velocity is achieved when the applied force is balanced by a resisting force (shown as an equal but opposite force applied by the stationary bottom plate).

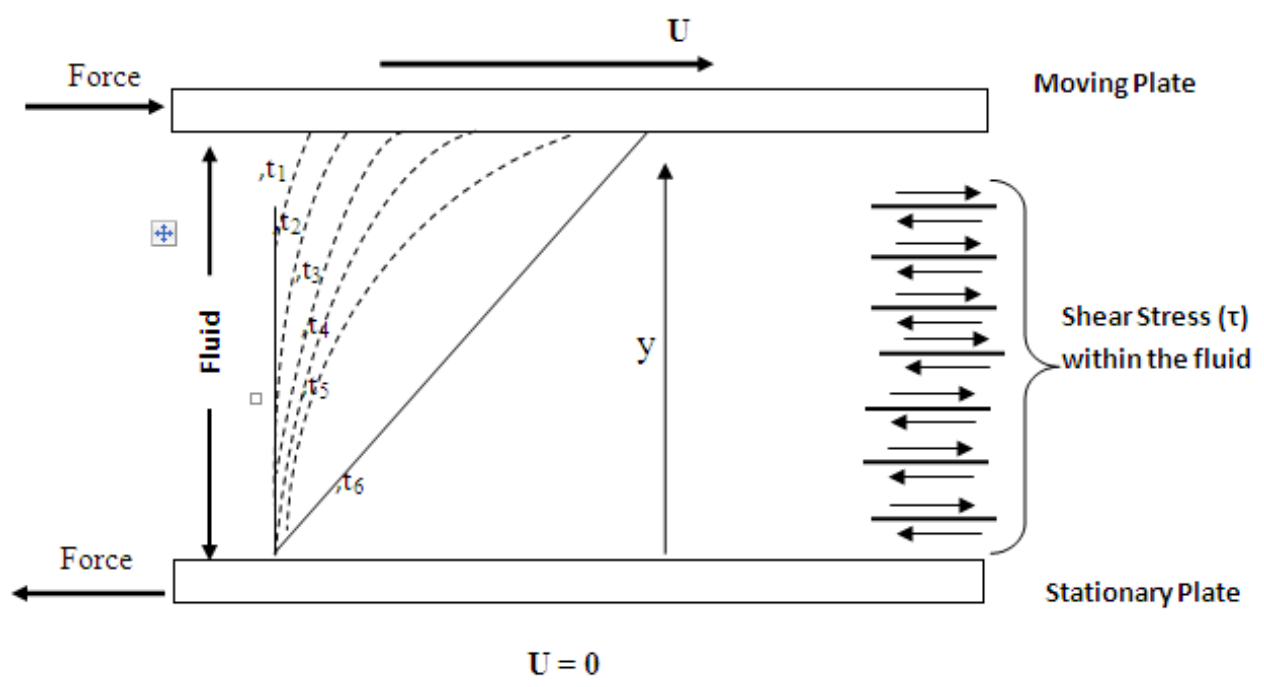

Fig. 1. Varying forces acting on flowing water along the flow depth

The shear stress transfers momentum (mass times velocity) through the fluid to maintain the linear velocity profile. The magnitude of the shear stress is equal to the force that is applied to the top plate. The relationship between the shear stress, the fluid viscosity and the velocity gradient is given by:

$$
\tau=\mu \frac{d u}{d y}
$$


Where $\mathrm{u}$ is the velocity, $\mathrm{y}$ is the fluid depth at this point as given in figure, $\mu$ is the fluid viscosity, and $\tau$ is the shear stress.

From this relationship we can determine the velocity at any point within the column of fluid. Rearranging the terms:

$$
\tau / \mu=\frac{d u}{d y} \quad \text { or } \int(\tau / \mu) d y=\int d u \quad \text { or } \quad u_{y}=(\tau / \mu) y+c
$$

where $c$ (the constant of integration) is the velocity at $y=0$ (where $u=0$ ) such that:

$$
u_{y}=\frac{\tau}{\mu} y
$$

From this relationship we can see the following:

a. That the velocity varies in a linear fashion from 0 at the bottom plate $(y=0)$ to some maximum at the highest position (i.e., at the top plate).

b. That as the applied force (equal to $\tau$ ) increases so does the velocity at every point above the lower plate.

c. That as the viscosity increases the velocity at any point above the lower plate decreases. Driving force is only the force applied to the upper, moving plate, and the shear stress (force per unit area) within the fluid is equal to the force that is applied to the upper plate. Fluid momentum is transferred through the fluid due to viscosity.

\section{Fluid gravity flows}

Water flowing down a slope in response to gravity e.g. in rivers, the driving force is the down slope component of gravity acting on the mass of fluid; more complicated because the deeper into the flow the greater the weight of overlying fluid. In reference to Figure 2 that shows the variation in velocity along the flowing water, $\mathrm{D}$ is the flow depth and $y$ is some height above the boundary, FG is the force of gravity acting on a block of fluid with dimensions, (D-y) $\times 1 \times 1$; here $y$ is the height above the lower boundary, $\theta$ is the slope of the water surface, it may be noted here that the depth is uniform so that this is also the slope of the lower boundary, and $\tau_{\mathrm{y}}$ is the shear stress that is acting across the bottom of the block of fluid and it is the down slope component of the weight of fluid in the block at some height y above the boundary.

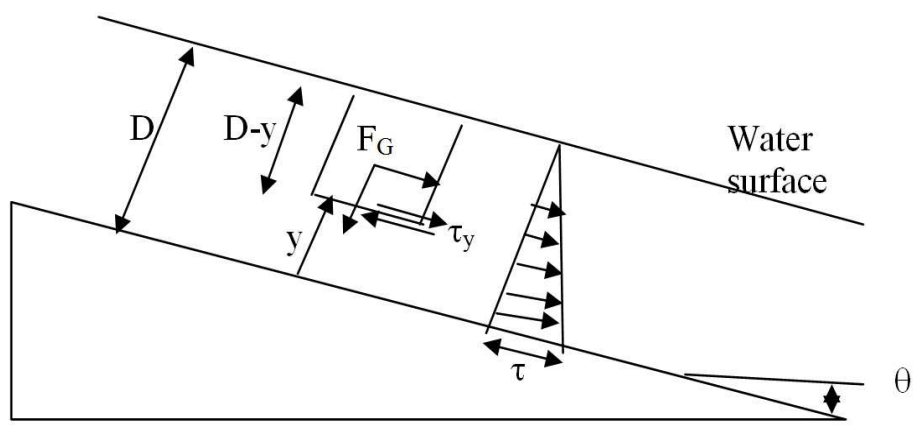

Fig. 2. Variation in velocity for depth 
For this general situation, $\tau_{\mathrm{y}}$, the shear stress acting on the bottom of such a block of fluid that is some distance $y$ above the bed can be expressed as follows:

$$
\tau_{y}=\rho g(D-y) \times 1 \times 1 \times \sin (\theta)
$$

The first term in the above equation i.e. $\rho g(D-y) \times 1 \times 1$ is the weight of water in the block and $\operatorname{Sin}(\theta)$ is the proportion of that weight that is acting down the slope. Clearly, the deeper within the water i.e. with decreasing $y$ the greater the shear stress acting across any plane within the flow. At the boundary $y=0$, the shear stress is greatest and is referred to as the boundary shear stress $\left(\tau_{0}\right)$; this is the force per unit area acting on the bed which is available to move sediment.

$$
\text { Setting } y=0: \quad \tau_{0}=\rho g(D-y) \sin (\theta) \text { and } \tau_{y}=\mu \frac{d u}{d y}
$$

From the above equations, we get the following velocity distribution for such flows by substituting

$$
d u / d y=\rho g(D-y) \sin (\theta) / \mu
$$

Integrating with respect to $y$ :

$$
u_{y}=\int \frac{d u}{d y} d y=\frac{\rho g \operatorname{Sin} \theta}{\mu} \int(D-y) d y+c=\int \frac{\rho g \operatorname{Sin} \theta}{\mu}\left(y D-y^{2} / 2\right)+c
$$

Where $\mathrm{c}$ is the constant of integration and equal to the velocity at the boundary $(\mathrm{Uy}=0)$ such that:

$$
u_{y}=\frac{\rho g \sin \theta}{\mu}\left(y D-\frac{y^{2}}{2}\right)
$$

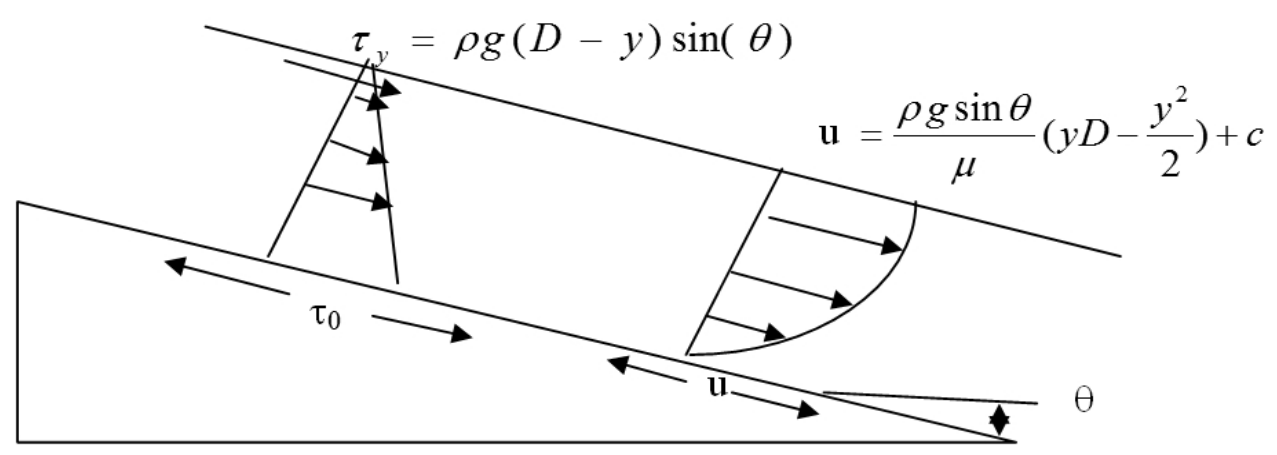

Fig. 3. Variation in velocity for depth

Velocity varies as an exponential function from 0 at the boundary to some maximum at the water surface; this relationship applies to: 
a. Steady flows: not varying in velocity or depth over time.

b. Uniform flows: not varying in velocity or depth along the channel.

c. Laminar flows: see next section.

\subsection{The classification of fluid gravity flows}

\subsubsection{Flow Reynolds' Number (R)}

Reynolds's experiments involved injecting a dye streak into fluid moving at constant velocity through a transparent tube. Fluid type, tube diameter and the velocity of the flow through the tube were varied, and the three types of flows that were classified are as follows: (a) Laminar Flow: every fluid molecule followed a straight path that was parallel to the boundaries of the tube, (b) Transitional Flow: every fluid molecule followed wavy but parallel path that was not parallel to the boundaries of the tube, and (c) Turbulent Flow: every fluid molecule followed very complex path that led to a mixing of the dye. Reynolds's combined these variables into a dimensionless combination now known as the Flow Reynolds' Number (R) where:

$$
R=\frac{\rho U D}{\mu}
$$

Where $U$ is the velocity of the flow, $\rho$ is the density of the fluid, $D$ is the diameter of the tube, and $\mu$ is the fluid's dynamic viscosity. Flow Reynolds' number is often expressed in terms of the fluid's kinematic viscosity ( $v$ ) equally expressed as $\mu / \rho$ (units are $\mathrm{m}^{2} / \mathrm{s}$ ) and

$$
R=\frac{U D}{v}
$$

The value of $\mathrm{R}$ determine the type of flows in the following manner:
a. Laminar flows:
$\mathrm{R}<1000$
b. Transitional flows:
$1000<\mathrm{R}<2000$
c. Turbulent flows:
$\mathrm{R}>2000$
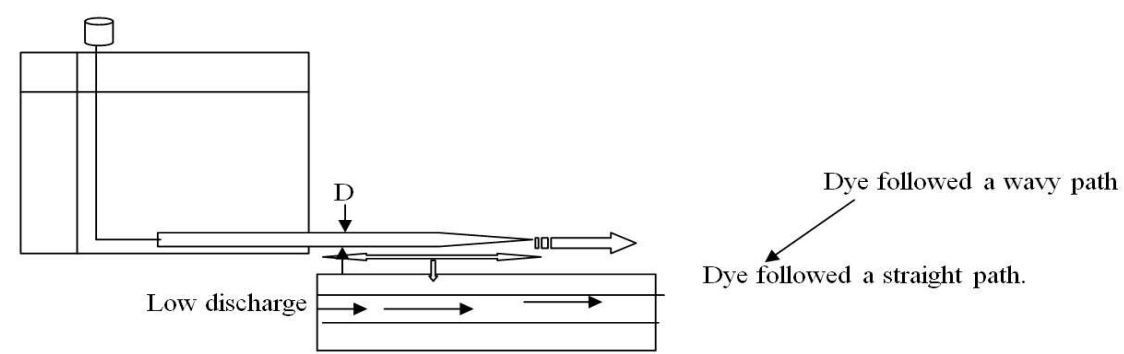

Medium discharge

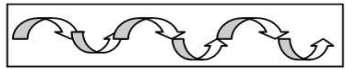

High discharge

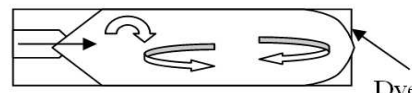

through the fluid in the tube

Fig. 4. Reynolds's experiments for different types of flows 
In laminar flows, the fluid momentum is transferred only by viscous shear; a moving layer of fluid drags the underlying fluid along due to viscosity (see the left diagram, below). The velocity distribution in turbulent flows has a strong velocity gradient near the boundary and more uniform velocity (an average) well above the boundary. The more uniform distribution well above the boundary reflects the fact that fluid momentum is being transferred not only by viscous shear. The chaotic mixing that takes place also transfers momentum through the flow. The movement of fluid up and down in the flow, due to turbulence, more evenly distributes the velocity, low speed fluid moves upward from the boundary and high speed fluid in the outer layer moves upward and downward. This leads to a redistribution of fluid momentum.

\section{Water Surface}

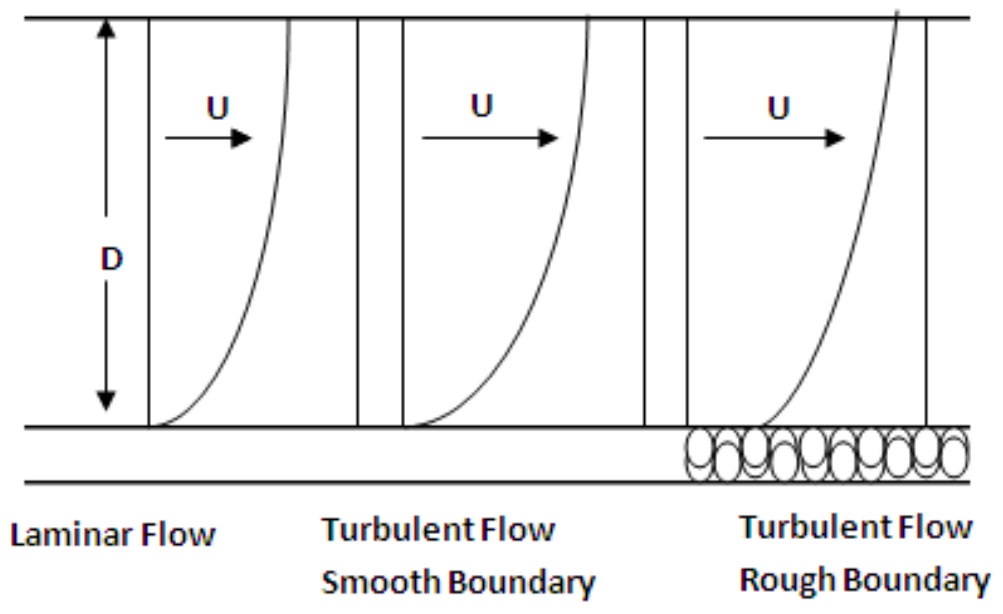

Fig. 5. Variation in velocity for depth at three different types of flows

Turbulent flows are made up of two regions. And there is an inner region near the boundary that is dominated by viscous shear i.e.,

$$
\tau_{y}=\mu \frac{d u}{d y}
$$

And, an outer region that is dominated by turbulent shear which focus on transfer of fluid momentum by the movement of the fluid up and down in the flow.

$$
\tau_{y}=\eta \frac{d u}{d y}+\mu \frac{d u}{d y}
$$

Where $\eta$ is the eddy viscosity which reflects the efficiency by which turbulence transfers momentum through the flow. 


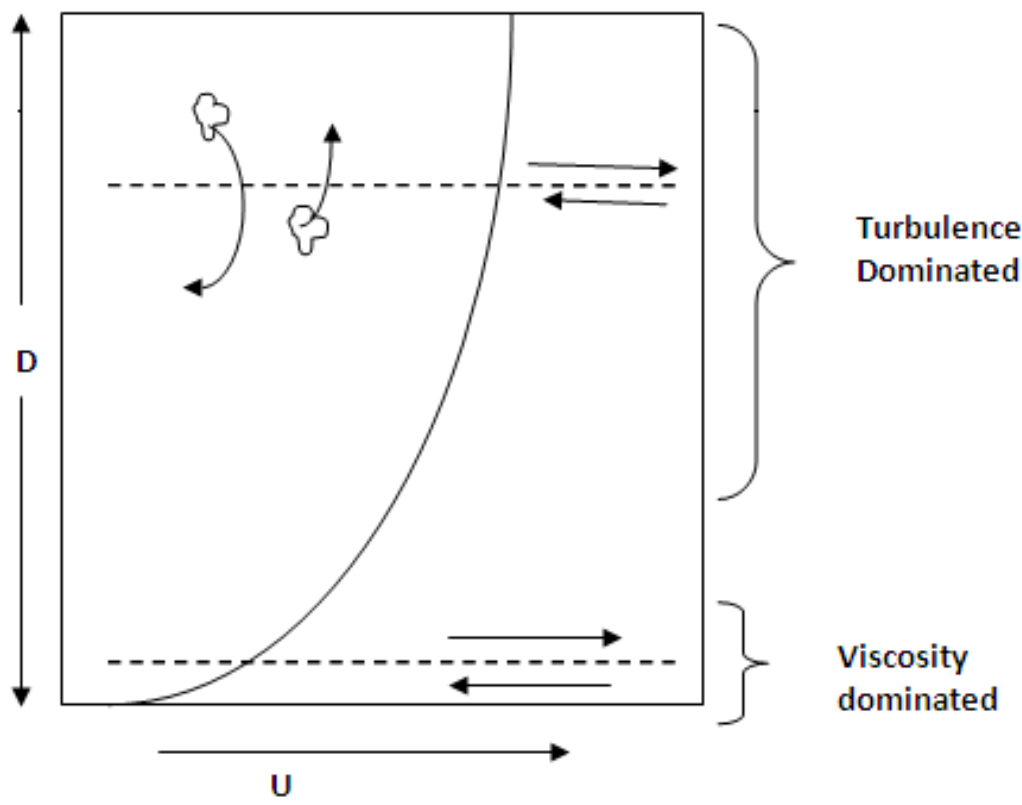

Fig. 6. Two regions of turbulent shear

As a result, the formula for determining the velocity distribution of a laminar flow cannot be used to determine the distribution for a turbulent flow as it neglects the transfer of momentum by turbulence. Experimentally, determined formulae are used to determine the velocity distribution in turbulent flows e.g. the Law of the Wall for rough boundaries under turbulent flows:

$$
\frac{u_{y}}{U_{*}}=8.5+\frac{2.3}{\kappa} \log \frac{y}{y_{0}} ; y_{0}(=\mathrm{d} / 30), U_{*}=\sqrt{\tau_{0 / \rho}} \text { and } \tau_{0}=\rho g D \operatorname{Sin}(\theta)
$$

Where $\kappa$ is Von Karman's constant which is generally taken 0.41 for clear water flows lacking sediment, $y$ is the height above the boundary, $\mathrm{y}_{0}(=\mathrm{d} / 30)$ and $\mathrm{d}$ is grain size, and $\mathrm{U}^{*}$ is the shear velocity of the flow. If the flow depth and shear velocity are known, as well as the bed roughness, this formula can be used to determine the velocity at any height $y$ above the boundary.

$$
\begin{gathered}
u_{y}=U_{*}\left(8.5+\frac{2.3}{\kappa} \log \frac{y}{y_{0}}\right) \\
u_{y}=U_{*}\left(8.5+\frac{2.3}{\kappa} \log \right) g D \operatorname{Sin}(\theta)
\end{gathered}
$$

The above formula may be used to estimate the average velocity of a turbulent flow by setting $y$ to 0.4 times the depth of the flow i.e. $y=0.4 \mathrm{D}$. Experiments have shown that the average velocity is at $40 \%$ of the depth of the flow above the boundary. 


\subsubsection{Flow Froude Number (F)}

Classification of flows according to their water surface behaviour, is an important part of the basis for classification of flow regime
a. $\mathrm{F}<1$
b. $\quad F=1$
has a sub critical flow (tranquil flow)
c. $\mathbf{F}>1$
has a critical flow

Flow Froude Number $(\mathrm{F})$ is defined as follow:

$$
F=\frac{U}{\sqrt{g D}}
$$

$\sqrt{g D}=$ the celerity (speed of propagation) of gravity waves on a water surface.

$\mathbf{F}<\mathbf{1}, \mathbf{U}<\sqrt{g D}$ : water surface waves will propagate upstream because they move faster than the current. Bed forms are not in phase with the water surface.

$\mathbf{F}>\mathbf{1}, \mathbf{U}>\sqrt{g D}$ : water surface waves will be swept downstream because the current is moving faster than they can propagate upstream. Bed forms are in phase with the water surface.

In sedimentology the Froude number, is important to predict the type of bed form that will develop on a bed of mobile sediment.

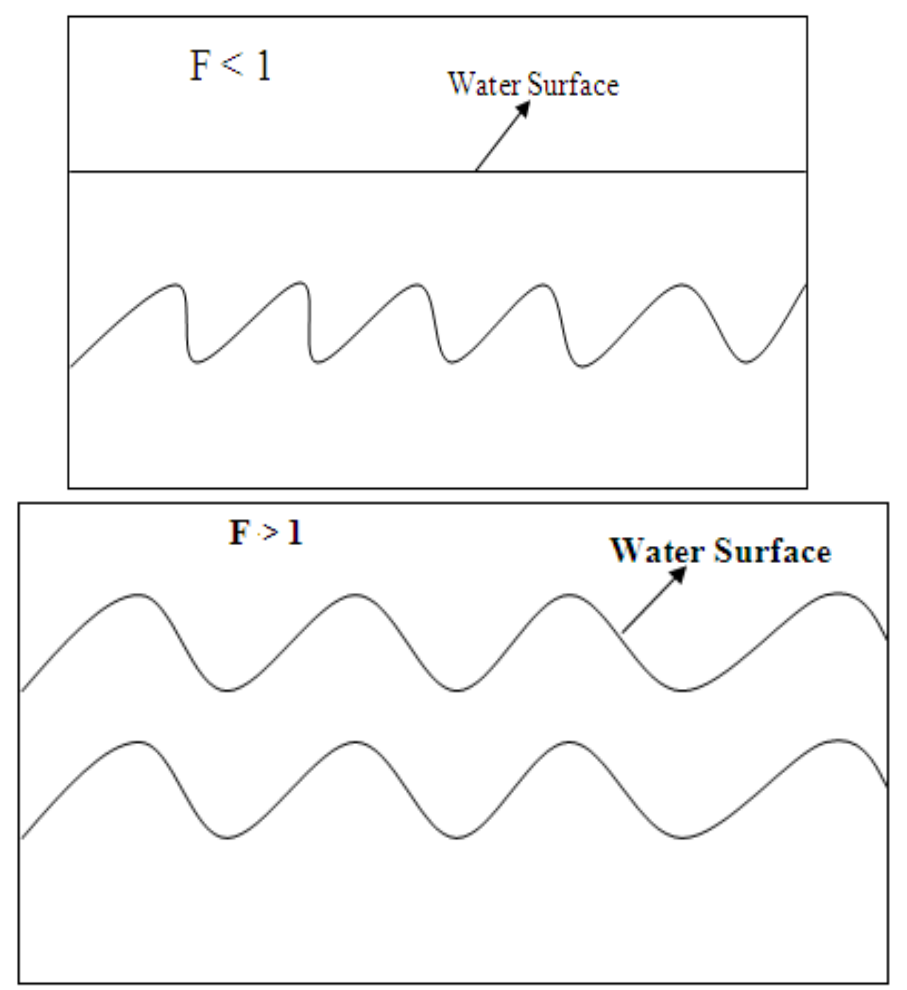

Fig. 7. Classification of flows according to degree of Froude Number 


\subsection{Velocity distribution, in turbulent flows}

Earlier we saw that for laminar flows the velocity distribution could be determined from Eq. (4). Eq. (8). Fig. 7 shows the turbulent flows and the corresponding two regions. As per the Law of the Wall for rough boundaries under turbulent flow depth, the shear velocity are known along with the bed roughness, and in such cases Eq. (10) can be used to determine the velocity at any height $y$ above the boundary.

\subsection{Subdivisions of turbulent flows}

Turbulent flows can be divided into three layers: (i) Viscous Sub layer is the region near the boundary that is dominated by viscous shear and quasi-laminar flow which is also referred to, inaccurately, as the laminar layer, (ii) Transition Layer lies intermediate between quasilaminar and fully turbulent flow, and (iii) Outer Layer which is fully turbulent and momentum transfer is dominated by turbulent shear.

\subsection{Viscous sub layer (VSL)}

The thickness of the VSL $(\delta)$ is known from experiments to be related to the kinematic viscosity and the shear velocity of the flow by:

$$
\delta=\frac{12 v}{U_{*}}
$$

It ranges from a fraction of a millimetre to several millimetres thick, and the thickness of the VSL particularly important in comparison to size of grains (d) on the bed. Next it shall be discussed about the forces that act on the grains and the variation of these relationships. The Boundary Reynolds' Number $\left(\mathbf{R}^{*}\right)$ is used to determine the relationship between $\delta$ and $\mathrm{d}$ :

$$
R_{*}=\frac{U_{*} D}{v}
$$

A key question is at what value of $R$ is the diameter of the grains on the bed equal to the thickness of the VSL?

Given that $\delta=\frac{12 v}{U_{*}}$, the condition exists when $\delta=\mathrm{d}$, and by substituting this relationship in $\mathrm{R}_{*}$

$R_{*}=\frac{U_{*} D}{v}=12$, thus

$\mathrm{R}^{*}<12 \delta>\mathrm{d}$

$\mathrm{R}^{*}=12 \mathrm{~d}=\mathrm{d}$

$\mathrm{R}^{*}>12 \quad \delta<\mathrm{d}$

Turbulent boundaries are classified on the basis of the relationship between thickness of the VSL and the size of the bed material. Given that there is normally a range in grain size on the boundary, the following shows the classification (Fig. 8):

At the boundary of a turbulent flow the average boundary shear stress $\left(\tau_{\mathrm{o}}\right)$ can be determined using the same relationship, as for a laminar flow. In the viscous sub layer viscous shear predominates so that the same relationship exists, as given in Eqs. (3a, 8 and 9) that applies to steady, uniform turbulent flows.

Boundary shear stress governs the power of the current to move sediment; specifically, erosion and deposition depend on the change in boundary shear stress in the downstream 
direction. In general, sediment transport rate $\left(q_{s}\right)$ is the amount of sediment that is moved by a current that increases with increasing boundary shear stress. When $\tau_{\mathrm{o}}$ increases downstream, so does the sediment transport rate; this leads to erosion of the bed providing that a $\tau_{\mathrm{o}}$ that is sufficient to move the sediment. When $\tau_{\mathrm{o}}$ decreases along downstream, so does the sediment transport rate; this leads to deposition of sediment on the bed. Variation in $\tau_{\mathrm{o}}$ along the flow due to turbulence leads to a pattern of erosion and deposition on the bed of a mobile sediment. This phenomena is given in Fig. 9.

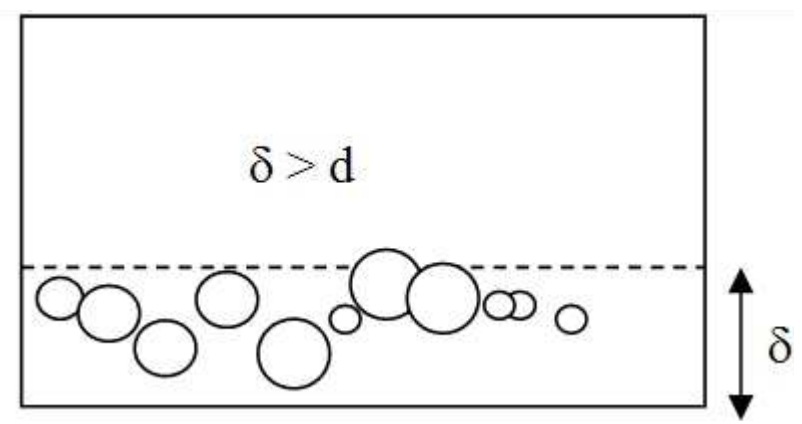

(a) For $\mathrm{R}^{*}<5$ is smooth

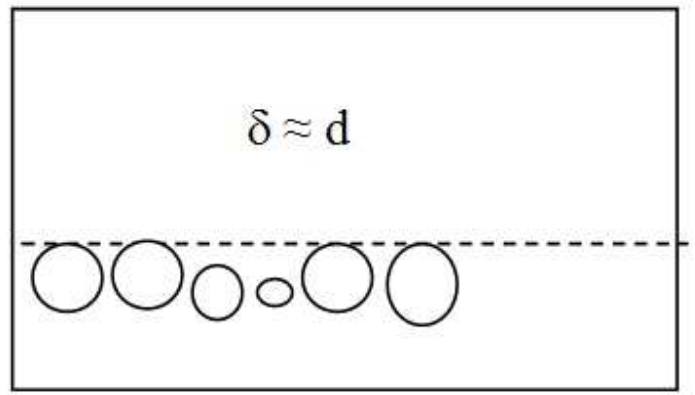

(b) For $5<\mathrm{R}^{*}<70$ is transitional

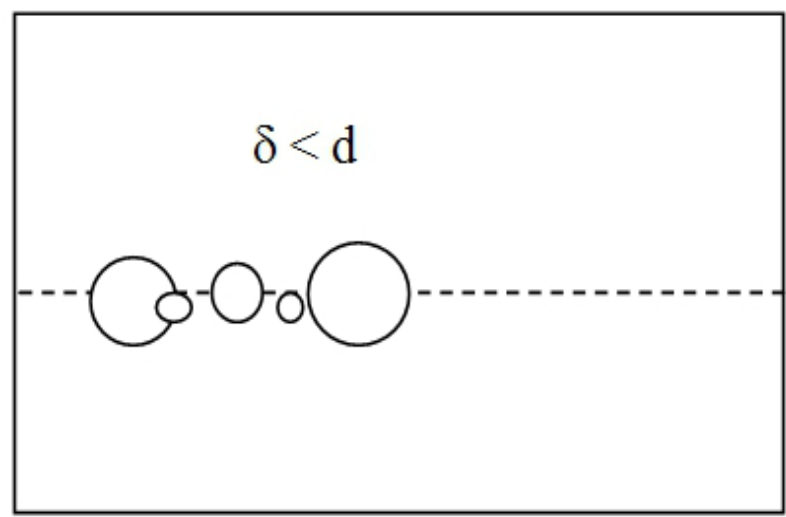

(c) For $R^{*}>70$ is Rough

Fig. 8. Classification of flows according to degree of Boundary Reynolds' Number 


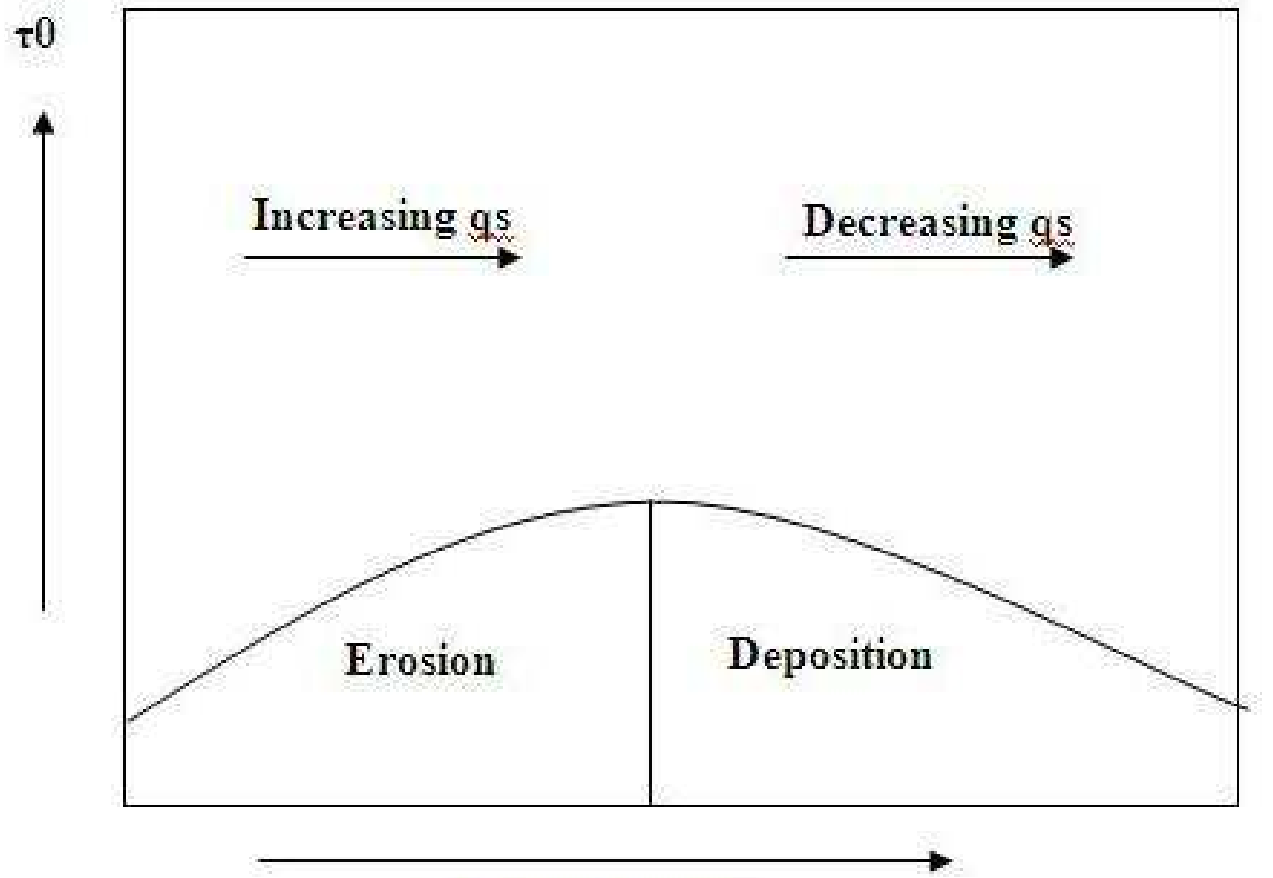

\section{Flow Direction}

Fig. 9. Pattern of bed erosion and deposition according to variation of shear stress.

\subsubsection{Large scale structures of the outer layer}

Secondary flows involves a rotating component of the motion of fluid about an axis that is parallel to the mean flow direction. Commonly there are two or more such rotating structures extending parallel to each other.

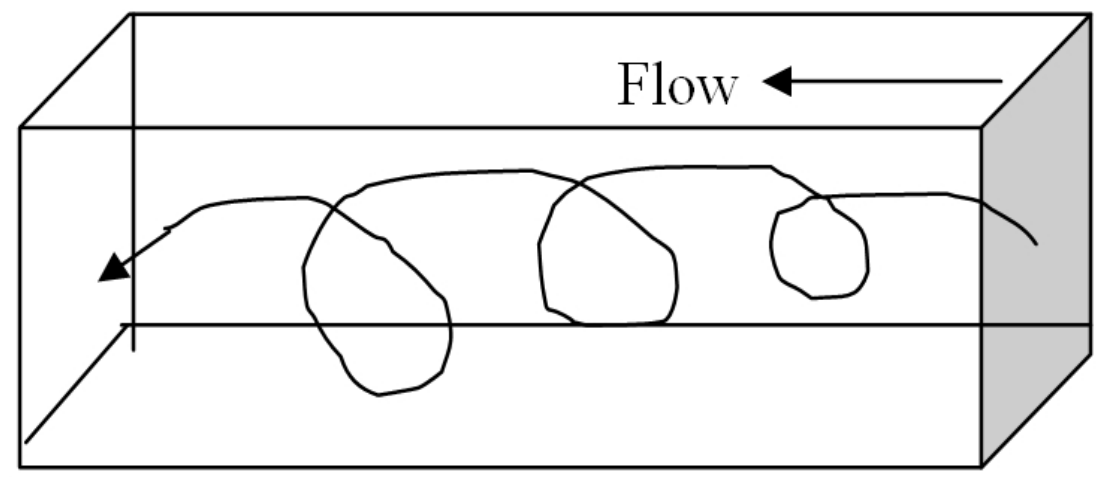

Fig. 10. Eddies about the axes perpendicular to the flow direction. 
In meandering channels, characterized by a sinusoidal channel form, counter-rotating spiral cells alternate from side to side along the channel. Eddies are components of turbulence that rotate about axes that are perpendicular to the mean flow direction. Smaller scale than secondary flows moves downstream with the current at a speed of approximately $80 \%$ of the water surface velocity $\left(\mathrm{U}_{\infty}\right)$. Eddies move up and down within the flow as the travel downstream, and this lead to variation in boundary shear stress over time and along the flow direction. Some eddies are created by the topography of the bed. In the lee of a negative step on the bed (see figure below) the flow separates from the boundary (" $s$ " in the figure) and reattaches downstream ("a" in the figure). A roller eddy develops between the point of separation and the point of attachment. Asymmetric bed forms (see next chapter) develop similar eddies.

(a)

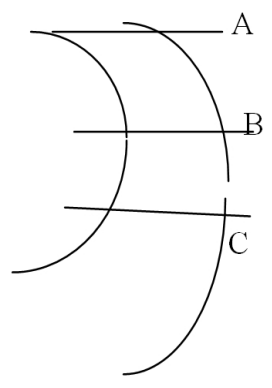

Fig. 11. Asymmetric bed forms
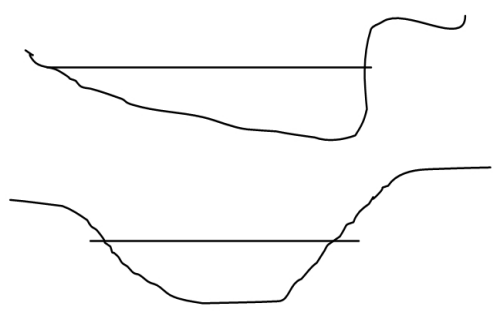

(b)

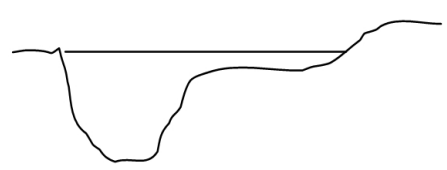

\subsubsection{Small scale structures of the viscous sub layer}

Alternating lanes of high and low speed fluid within the VSL are termed as streaks associated with counter-rotating, flow parallel vortices within the VSL. Streak spacing $(\lambda)$ varies with the shear velocity $\left(U_{*}\right)$ and the kinematic viscosity $(v)$ of the fluid; $\lambda$ ranges from millimetres to centimetres. The relationship is as follows:

$$
\lambda=\frac{100 v}{U_{*}}
$$

$\lambda$ increases when sediment is present. Due to fluid speed, a bursting cycle is referred as:

Burst: ejection of low speed fluid from the VSL into the outer layer.

Sweep: injection of high speed fluid from the outer layer into the VSL.

Often referred to as the bursting cycle but not every sweep causes a burst and vise versa, however, the frequency of bursting and sweeps are approximately equal.

\subsection{Sediment transport under unidirectional flows}

The sediment that is transported by a current comes under two main classes:

Wash load: silt and clay size material that remains in suspension even during low flow events in a river. 
Bed material load: sediment (sand and gravel size) that resides in the bed but goes into transport during high flow events e.g., floods.

Bed material load makes up many arsenates and ratites in the geological record. Three main components of bed material load are: Contact load: particles that move in contact with the bed by sliding or rolling over it. Saltation load: movement as a series of hops along the bed, each hop following a ballistic trajectory.

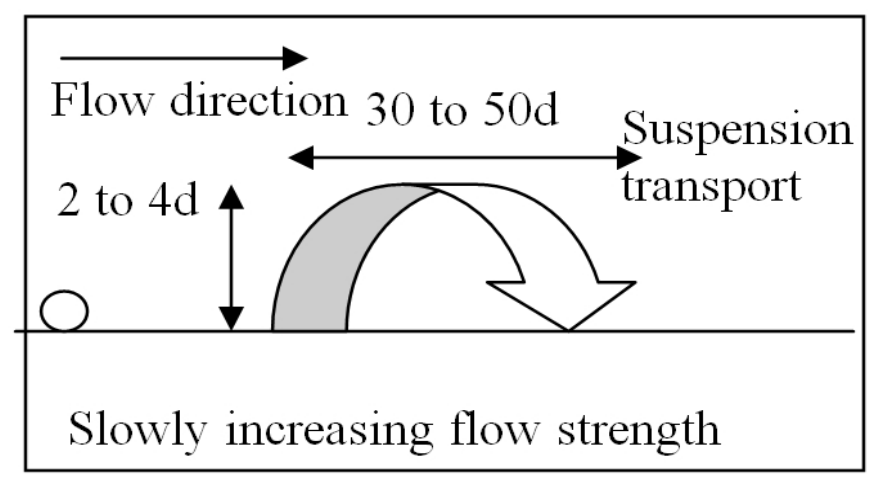

Fig. 12. The ballistic trajectory in the flow

When the ballistic trajectory is disturbed by turbulence, the motion is referred to as Suspensive saltation.

Intermittent suspension load: carried in suspension by turbulence in the flow. Intermittent because it is in suspension only during high flow events, and otherwise, resides in the deposits of the bed. Bursting is an important process in initiating suspension transport.

\subsection{Hydraulic interpretation of grain size distributions}

In the section on grain size distributions we saw that some sands are made up of several normally distributed sub-populations. These sub-populations can be interpreted in terms of the modes of transport that they underwent prior to deposition. The finest sub-population represents the wash load. Only a very small amount of wash load is ever stored within the bed material so that it makes up a very small proportion of these deposits. The coarsest subpopulation represents, the contact and saltation loads. In some cases they make up two subpopulations (only one is shown in the Fig.13).

The remainder of the distribution, normally making up the largest proportion, is the intermittent suspension load. This interpretation of the subpopulations gives us two bases for quantitatively determining the strength of the currents that transported the deposits. The grain size $X$ is the coarsest sediment that the currents could move on the bed. In this case, $X$ $=-1.5 \phi$ or approximately $2.8 \mathrm{~mm}$. If the currents were weaker, that grain size would not be present. And, if the currents were stronger, coarser material would be present. This assumes that there are no limitations to the size of grains available in the system. The grain size $Y$ is the coarsest sediment that the currents could take into suspension. In this case, $Y=1.3 \mathrm{f}$ or 
approximately $0.41 \mathrm{~mm}$, therefore the currents must have been just powerful enough to take the $0.41 \mathrm{~mm}$ particles into suspension. If the currents were stronger the coarsest grain size would be larger. This follows the above assumption of limitations to the size of grains size in a system.

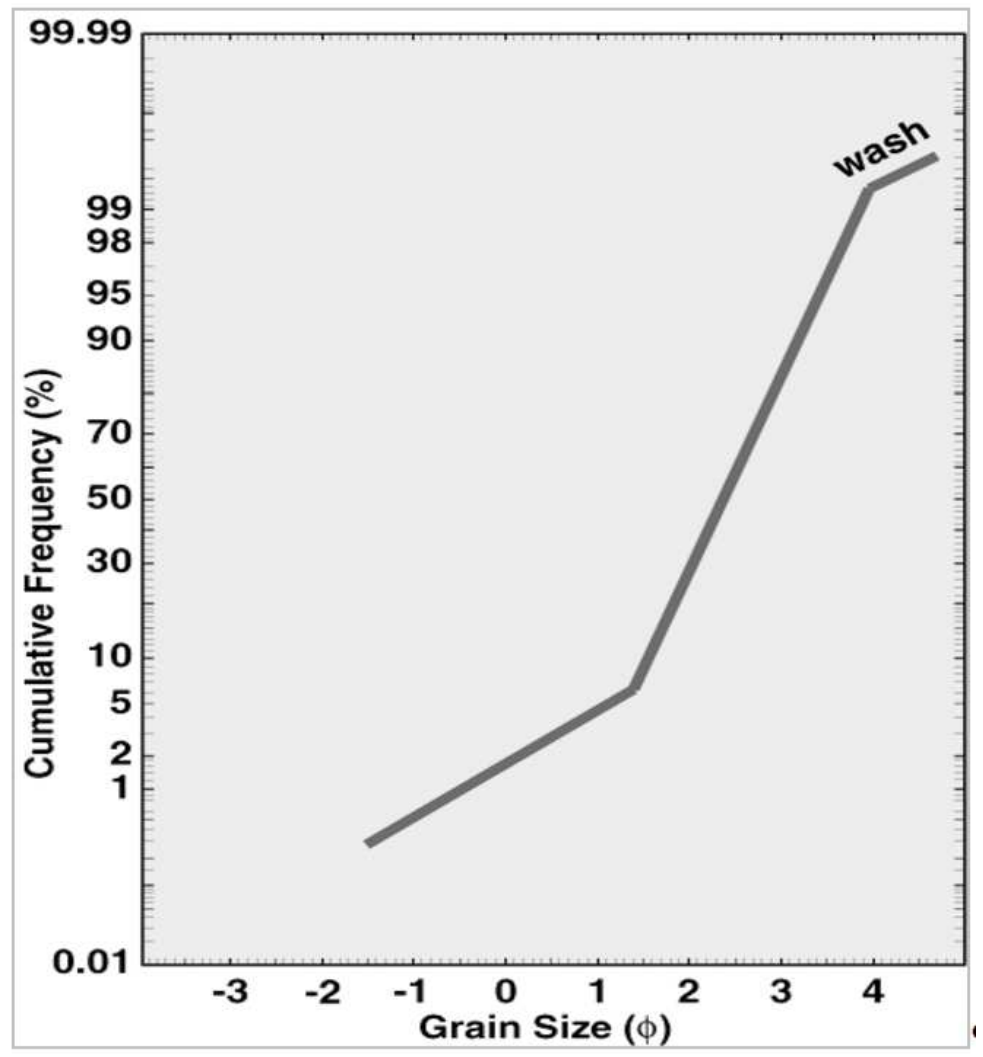

Fig. 13. The grain size frequency distribution

To quantitatively interpret $X$, we need to know the hydraulic conditions needed to just begin to move of that size. This condition is the threshold for sediment movement. To quantitatively interpret $Y$ we need to know the hydraulic conditions needed to just begin carry that grain size in suspension. This condition is the threshold for suspension.

\subsection{The threshold for grain movement on the bed}

Grain size $X$ can be interpreted, if we know what flow strength is required to just move a particle of that size. That flow strength will have transported sediment with that maximum 
grain size. Several approaches have been taken to determine the critical flow strength to initiate motion on the bed.

Hjulstrom's Diagram shows the diagram of the critical velocity that is required to just begin to move sediment of a given size i.e. the top of the mud region. It also shows the critical velocity for deposition of sediment of a given size at the bottom of the field. The experiment is based on a series of experiments using unidirectional currents with a flow depth of $1 \mathrm{~m}$. It can be noted here that for grain sizes coarser than $0.5 \mathrm{~mm}$ the velocity that is required for transport increases with grain size; the larger the particles the higher velocity the is required for transport. For finer grain sizes (with cohesive clay minerals), the greater the critical velocity for transport. This is because the more mud is present means that the cohesion is greater, and the resistance to erosion increases, despite the finer grain size. In our example, the coarsest grain size was $2.8 \mathrm{~mm}$. According to Hjulstron's diagram that grain size would require a flow with a velocity of approximately $0.65 \mathrm{~m} / \mathrm{s}$. Therefore, the sediment shown in the cumulative frequency curve, was transported by currents at $0.65 \mathrm{~m} / \mathrm{s}$.

The problem is that the forces that are required to move sediment, are not only related to flow velocity, but also the boundary shear stress that is a significant force. Boundary shear stress varies with flow depth, as shown the relationship earlier given in Eq. (9) as $\tau_{0}=\rho g D \operatorname{Sin}(\theta)$. Therefore, Hjulstrom's diagram is reasonably accurate only for sediment that has been deposited under flow depths of $1 \mathrm{~m}$.

\subsection{Shield's criterion for the initiation of motion}

Based on a large number of experiments Shield's criterion considers the problem in terms of the forces that act to move a particle. The criterion applies to beds of spherical particles of uniform grain size. Forces that are important to initial motion are as follows:

1. The submerged weight of the particle can be taken as $\pi / 6\left(\rho_{\mathrm{s}}-\rho\right) \mathrm{g} \mathrm{d}^{3}$ which resists motion.

2. To which causes a drag force that acts to move the particle down current

3. Lift force (L) that reduces the effective submerged weight.

The flow velocity that is felt by the particle varies from approximately zero at its base to some higher velocity at its highest point.

Pressure specifically dynamic pressure in contrast to static pressure is also imposed on the particle and the magnitude of the dynamic pressure varies inversely with the velocity. For, higher velocity, lower dynamic pressure, and maximum dynamic pressure is exerted at the base of the particle and minimum pressure at its highest point. The dynamic pressure on the particle varies symmetrically from a minimum at the top to maximum at the base of the particle. As shown in Fig. 14, this distribution of dynamic pressure results in a net pressure force that acts upwards. Thus, the net pressure force known as the Lift Force acts opposite to the weight of the particle reducing its effective weight. This makes it easier for the flow to roll the particle along the bed. The lift force reduces the drag force that is required to move the particle. If the particle remains immobile to the flow and the velocity gradient is large enough so that the Lift force exceeds the particle's weight, it will jump straight upwards away from the bed. Once off the bed, the pressure difference from top to bottom of the particle is lost and it is carried down current as it falls back to the bed following the ballistic trajectory of saltation. 


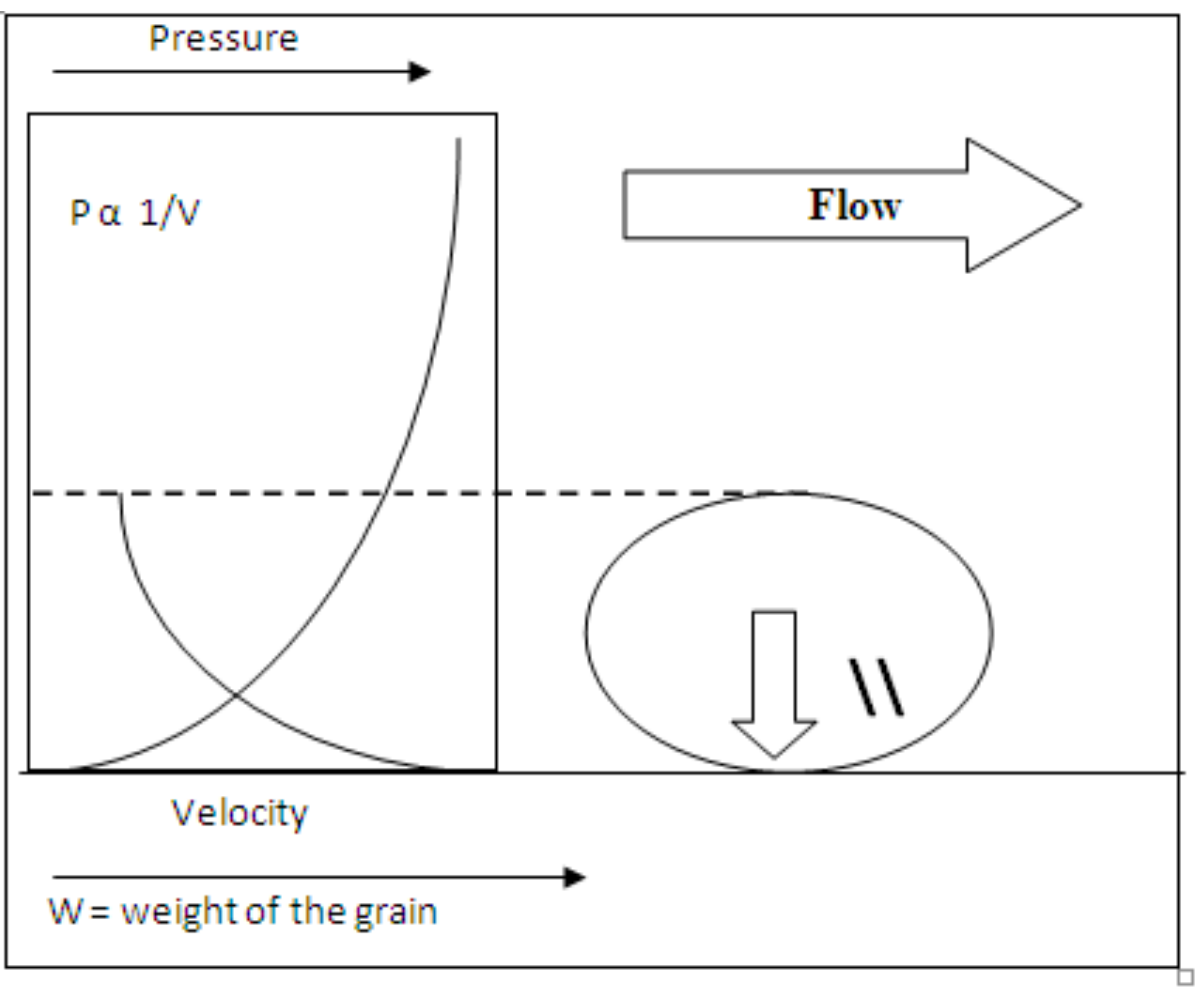

Fig. 14. Simplified ray diagram showing the forces required for initial motion

Shield's experiments involved determining the critical boundary shear stress required to move spherical particles of various size and density over a bed of grains with the same properties (uniform spheres). He produced a diagram that allows the determination of the critical shear stress required for the initiation of motion. A bivariate plot of "Shield's Beta" versus Boundary Reynolds' Number

$$
\beta=\frac{\tau_{0}}{\left(\rho_{s}-\rho\right) g d}=\text { (Force acting to move the particle excluding lift) } /
$$

(Force resisting movement)

$\tau_{0}$ is the critical shear stress for motion, and the denominator gives the submerged weight of grains per unit area on the bed. As the lift the force increases $\beta$ will decrease that shall lower required for movement. Reflects $R_{*}=\frac{U_{*} d}{v}$ something of the lift force (related to the velocity gradient across the particle).

For low boundary Reynold's numbers Shield's $\beta$ decreases with increasing $R^{*}$ (Fig. 15). For high boundary Reynold's numbers Shield's $\beta$ increases with increasing $R^{*}$. The change takes place at $R^{*} \approx 12$. 


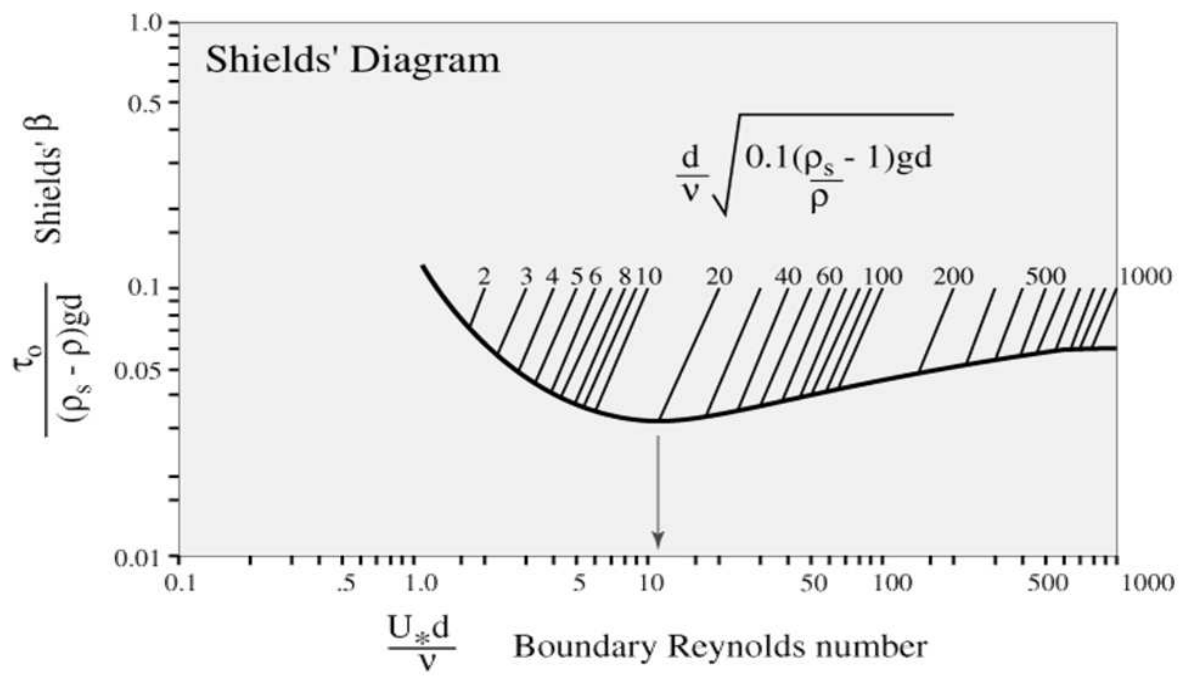

Fig. 15. Shield's Diagram
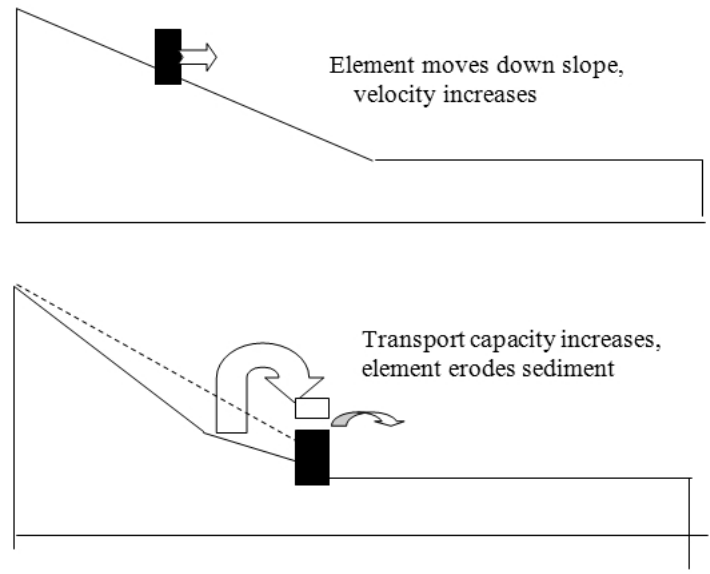

As velocity decreases, transport capacity decreases, element deposits sediment

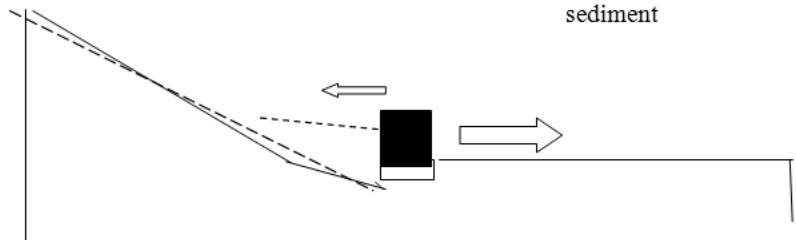

Fig. 16. Two dimensional flow simulation with flow depth 
The upstream boundary condition needed to route sediment through a network of stream channels, there is no established method exists for a specific watershed. An example is illustrated in Fig. 17.
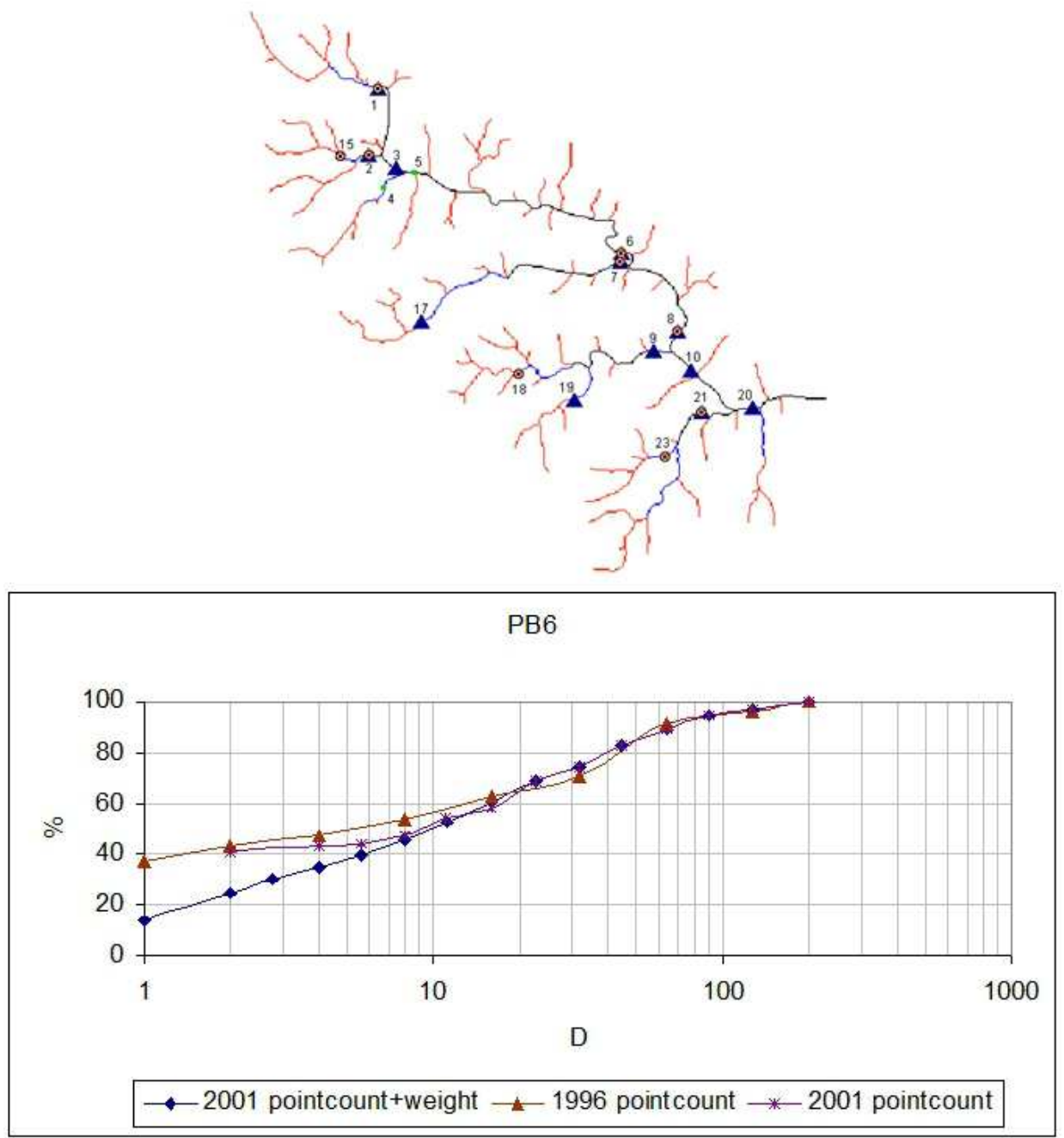

Fig. 17. Regression equations relating sediment grain size distribution of the bed and bank sediment throughout a \% of the basin over decadal timescales

\section{Sediment transport}

This is the movement of solid particles and sediment is naturally-occurring material that is broken down by processes of weathering and erosion, and is subsequently transported by the action of fluids such as wind, water, or ice and/or by the force of gravity acting on the 
particle itself , typically due to a combination of the force of gravity acting on the sediment and/or the movement of the fluid. A fluid is a substance that continually deforms under an applied shear stress, no matter how small it is. In general, fluids are a subset of the phases of matter and include liquids, gases, plasmas and, to some extent, plastic solids in which the sediment is entrained. An understanding of sediment transport is typically used in natural systems, where the particles are elastic rocks.

The estimation of sediment yield is needed for studies of reservoir sedimentation, river morphology, and soil and water conservation planning. However, sediment yield estimate of a watershed is difficult as it results due to a complex interaction between topographical, geological, and soil characteristics. Sediment graph provides useful information to estimate sediment yield to study transport of pollutants attached to the sediment. To determine these sediment graphs, simple conceptual models are used, which are based on spatially lumped form of continuity and linear storage-discharge equations. Here a watershed is represented by storage systems that include the catchment processes, without including the specific details of process interactions. Examples of few conceptual models are given by (RendonHerrero, 1978; Williams, 1978; Singh et al., 1982; Chen and Kuo, 1984; Kumar and Rastogi, 1987; and Lee and Singh, 2005). Rendon-Herrero, (1978) defined the unit sediment graph (USG) resulting due to one unit of mobilized sediment for a given duration uniformly distributed over a watershed. Similarly, Williams (1978\} model is based on the instantaneous unit sediment graph (IUSG) concept, where IUSG was defined as the product of the IUH and the sediment concentration distribution (SCD), which was assumed to be an exponential function for each event and was correlated with the effective rainfall characteristics. In Chen and Kuo (1984) model the mobilized sediment was related regressionally with effective-rainfall, and rainfall records and watershed characteristics are to be known necessarily. A similar regression approach was followed by Kumar and Rastogi (1987), Raghuwanshi et al. (1994, 1996), and Sharma and Murthy (1996) to derive sediment graph and peak sediment flow rates from a watershed to reflect the respective changes due to land management practices. However, this routine procedure of regression between mobilized sediment and effective-rainfall always does not produce satisfactory results (Raghuwanshi et al., 1994, 1996). Moreover, the IUSG models utilizing the regression relationship for sediment graph derivation does not explicitly consider the major runoff and sediment producing characteristics of watershed i.e. soil, land use, vegetation and hydrologic condition in their formulation.

In addition to the above approaches discussed so far, the Soil Conservation Service Curve number (SCS-CN) method has also been used for sediment yield modeling (Mishra et al. 2006). Since the method is simple and well established in hydrologic, agriculture and environmental engineering, and is discussed here as it considers the effects of soil type, land use/treatment, surface condition, and antecedent condition. In a recent book by Singh and Frevert (2002), at least six of the twenty-two chapters present mathematical models of watershed hydrology that use the SCS-CN approach, and it shows a lot about the robustness of the SCS-CN methodology and its lasting popularity. Recently Mishra et al. (2006) developed sediment yield models using SCS-CN method, delivery ratio $\left(D_{R}\right)$ concept, and USLE. The models take care of various elements of rainfall-runoff process such as initial abstraction; initial soil moisture; and initial flush. However, the developed models are not applicable for estimation of sediment graphs (sediment flow rate versus time).

With the above back ground, the following sections discuss a simple sediment yield model based on SCS-CN method, Power law (Novotony and Olem, 1994), and utilizes linear 
reservoir concept similar to Nash (1960) to estimate sediment flow rates and total sediment yield as well. Briefly the model comprises of (i) the mobilized sediment estimation by SCSCN method and Power law (Novotony and Olem, 1994), instead of relating mobilized sediment and effective-rainfall regressionally; and (ii) the mobilized sediment is then routed through cascade of linear reservoirs similar to Nash (1960). The shape and scale parameters of the IUSG are determined from available storm sediment graphs and then direct sediment graphs are computed by convolution of the IUSG with mobilized sediment. It is noteworthy here that the model does not explicitly account for the geometric configuration of a given watershed.

\subsection{Mathematical formulation of proposed model}

The suspended sediment dynamics for a linear reservoir can be represented by a spatially lumped form of continuity equation and a linear-storage discharge relationship, as follows:

First linear reservoir:

$$
\begin{gathered}
I_{s 1}(t)-Q_{s 1}(t)=d S_{s 1}(t) / d t \\
S_{s 1}(t)=K_{s} Q_{s 1}(t)
\end{gathered}
$$

where $I_{s 1}(t)$ is the sediment inflow rate to the first reservoir [MT-1], and specified in units of (Tons/hr), $Q_{s 1}(t)$ is the sediment outflow rate [MT-1] in units of (Tons/hr), $S_{s 1}(t)$ is the sediment storage within the reservoir specified in Tons, and $K_{s}$ is sediment storage coefficient in hours..

For an instantaneous inflow i.e. $I_{s 1}(t)=0$, Eq. (16) converts to

$$
0-Q_{s 1}(t)=d S_{s 1}(t) / d t
$$

Substituting the value of $S_{s 1}(t)$ from Eq. (17) in Eq. (18), a simplified form of Eq. (18) is deduced as follows:

$$
0-Q_{s 1}(t)=d\left(K_{s} Q_{s 1}(t)\right)
$$

On rearranging Eq. (19) and performing integration operation one gets

$$
\begin{gathered}
\int d Q_{s 1}(t) / Q_{s 1}(t)=-\left(1 / K_{s}\right) \int d t \\
-t / K_{s}+C_{1}=\ln Q_{s 1}(t)
\end{gathered}
$$

where $C_{1}$ is the constant of integration. $C_{1}$ can be estimated by putting $t=0$ in Eq. (21) to get $C_{1}=-\ln Q_{s 1}(0)$, which on substituting in Eq. (21) and on rearranging gives

$$
Q_{s 1}(t)=Q_{s 1}(0) e^{-t / K_{s}}
$$

For $\mathrm{t}=0$, Eq. (17) reduces to

$$
S_{s 1}(0)=K_{s} Q_{s 1}(0)
$$


Defining $\mathrm{A}_{\mathrm{c}}$ as the watershed area in $\mathrm{Km}^{2}$ and $\mathrm{Y}$ as mobilized sediment per storm in Tons $/ \mathrm{km}^{2}$, the total amount of mobilized sediment $\mathrm{Y}_{\mathrm{T}}=\mathrm{A}_{\mathrm{c}} \mathrm{Y}$ Tons. If this much amount occurs instantaneously for one unit, i.e., $S_{s 1}(0)=A_{c} Y=1$, Eq. (23) simplified to the following form

$$
1=K_{s} Q_{s 1}(0)
$$

Coupling of Eqs. (22) \& (23) results,

$$
Q_{s 1(t)}=\left(1 / K_{s}\right) e^{-t / K_{s}}
$$

Eq. (25) gives nothing but the rate of sediment output from the first reservoir. This output forms the input to second reservoir and if it goes on up to $\mathrm{n}^{\text {th }}$ reservoir, then the resultant output from the $\mathrm{n}^{\text {th }}$ reservoir can be derived as:

$$
Q_{s n}(t)=\left[\left(t / K_{s}\right)^{n-1} e^{-t / K_{s}}\right] / K_{s} \Gamma(n)
$$

where $\Gamma()$ is the Gamma function. Eq. (26) represents the IUSG ordinates at time $\mathrm{t}\left(\mathrm{hr}^{-1}\right)$. For the condition, at $\mathrm{t}=\mathrm{t}_{\mathrm{p}}$ or $d Q_{s n}(t) / d t=0$, yields

$$
K_{s}=t_{p} /(n-1)
$$

Coupling of Eqs. (26) \& (27) yields

$$
Q_{s n}(t)=(n-1)^{n} / t_{p} \Gamma(n)\left[\left(t / t_{p}\right) e^{-\left(t / t_{p}\right)}\right]^{n-1}
$$

Eq. (28) gives the output of the $\mathrm{n}^{\text {th }}$ linear reservoir.

The SCS-CN method is based on the water balance equation and two fundamental hypotheses, which can be expressed mathematically, respectively, as:

$$
\begin{gathered}
P=I_{a}+F+Q \\
Q / P-I_{a}=F / S \\
I_{a}=\lambda S
\end{gathered}
$$

where, $\mathrm{P}$ is total precipitation, $\mathrm{I}_{\mathrm{a}}$ initial abstraction, $\mathrm{F}$ cumulative infiltration, $\mathrm{Q}$ direct runoff, $S$ potential maximum retention, and $\lambda$ initial abstraction coefficient. Combination of Eqs. (29) and (30) leads to the popular form of SCS-CN method, expressible as:

$$
\begin{array}{ll}
Q=\left(P-I_{a}\right)^{2} / P-I_{a}+S & \text { for } \mathrm{P}>\mathrm{I}_{\mathrm{a}} \\
=0 & \text { otherwise }
\end{array}
$$

Alternatively, for $I_{a}=0$, Eq. (32) reduces to

$$
\begin{array}{ll}
Q=P^{2} / P+S & \text { for } P>0 \\
=0 & \text { otherwise }
\end{array}
$$


Following Mishra and Singh (2003) for the condition, $\mathrm{f}_{\mathrm{c}}=0$, the Horton's method (Horton, 1938) can be expressed mathematically as:

$$
f=f_{0} e^{-k t}
$$

where $\mathrm{f}$ is the infiltration rate $\left(\mathrm{L} \mathrm{T}^{-1}\right)$ at time $t, \mathrm{f}_{\mathrm{o}}$ is the initial infiltration rate (LT-1) at time $t=0$, $k$ is the decay constant $\left(T^{-1}\right)$, and $f_{c}$ is the final infiltration rate (LT $\left.{ }^{-1}\right)$. The cumulative infiltration $\mathrm{F}$ can be derived on integrating Eq. (34) as:

$$
F=f_{0}\left(1-e^{-k t}\right) / k
$$

It can be observed from Eq. (35) that as $\mathrm{F} \rightarrow \mathrm{f}_{\mathrm{o}} / \mathrm{k}$, as $\mathrm{t} \rightarrow \infty$, Similarly, for Eq. (30) as $\mathrm{Q} \rightarrow(\mathrm{P}$ $\left.\mathrm{I}_{\mathrm{a}}\right), \mathrm{F} \rightarrow \mathrm{S}$, and time $\mathrm{t} \rightarrow \infty$, therefore the similarity between the two yields

$$
S=f_{o} / k
$$

On the basis of infiltration tests, Mein and Larson, (1971) got $f_{o}=i_{o}$, where $i_{o}$ is the uniform rainfall intensity when $t=0$. Substituting this into Eq. (36) yields

$$
f_{0}=i_{0}=k S
$$

Eq. (37) describes the relationship among the three parameters $f_{o}, k$, and $S$. Thus Eq. (37) shows that $\mathrm{k}$ depends on the magnitude of the rainfall intensity and soil type, land use, hydrologic condition, and antecedent moisture that affect $S$ and the results are consistent as reported by Mein and Larson (1971). An assumption that rainfall P linearly increases with time $\mathrm{t}$ leads to

$$
P=i_{0} t
$$

which is a valid and reasonable assumption for infiltration rate computation in experimental tests (Mishra and Singh, 2004). Coupling of Eqs. (37) \& (38) gives,

$$
\mathrm{P}=\mathrm{kSt}
$$

The Power law proposed by Novotony and Olem (1994) can be expressed as

$$
D_{R}=\alpha C_{r}^{\beta}
$$

where $C_{\mathrm{r}}=$ runoff coefficient; $\mathrm{D}_{\mathrm{R}}=$ sediment delivery ratio; $\alpha$ and $\beta=$ the coefficient and exponent of power relationship. The ratio, $D_{R}$, is dimensionless and is expressed in terms of Sediment yield $\mathrm{Y}$ and Potential maximum erosion A as follows:

$$
D_{R}=Y / A
$$

The coefficient, $\mathrm{C}$ is also dimensionless, and expressed in terms of $\mathrm{Q}$ and $\mathrm{P}$, as:

$$
C_{r}=Q / P
$$

Substituting the expressions of $D_{R}$ and $C_{r}$ in Eq. (40) one gets

$$
Y=\alpha A(Q / P)^{\beta}
$$


In general, the potential maximum erosion (A) for storm based applications is computed by MUSLE (Williams, 1975a) as:

$$
A=11.8\left(V_{Q} Q_{P}\right)^{0.56} K(L S) C P
$$

where $V_{Q}$ is the volume of runoff in $\mathrm{m}^{3}, \mathrm{Q}_{\mathrm{P}}$ is the peak flow rate in $\mathrm{m}^{3} / \mathrm{s}, \mathrm{K}$ is the soil erodibility factor, LS is the topographic factor, $\mathrm{C}$ is the cover and management factor and $\mathrm{P}$ is the support practice factor.

For the condition $\mathrm{I}_{\mathrm{a}}=0$, equating Eqs. (30) \& (32) reduces to

$$
Q / P=P /(P+S)=F / S
$$

Substituting the equality $Q / P=P /(P+S)$ (Eq. 45) in Eq. (43) results

$$
Y=\alpha A[P /(P+S)]^{\beta}
$$

Similarly, the coupling of Eqs. (24) \& (31) yields

$$
Y=\alpha A[k t /(1+k t)]^{\beta}
$$

Thus, Eq. (47) gives the expression for mobilized sediment due to an isolated storm event occurring uniformly over the watershed. Hence, total amount of mobilized sediment is expressed as:

$$
Y_{T}=\alpha A A_{c}[k t /(1+k t)]^{\beta}
$$

Finally, coupling of Eq. (48) results as follows:

$$
Q_{n s}(t)=\left[\alpha A_{c}[k t /(1+k t)]^{\beta}(n-1)^{n} / t_{p} \Gamma(n)\left[\left(t / t_{p}\right) e^{-\left(t / t_{p}\right)}\right]^{n-1}\right]
$$

The expression given by Eq. (49) is the proposed model for computations of sediment graphs. The proposed model has four parameters $\alpha, \beta, \mathrm{k}$, and $\mathrm{n}$.

\subsection{Application}

The workability of the proposed model is tested using the published data of Chaukhutia watershed of Ramganga Reservoir catchment (Kumar and Rastogi, 1987, Raghuwanshi et al., 1994, 1996), a schematic map of the watershed is given in Fig. 18. The basic characteristics of sediment graph data are given in Table 1.

\subsection{Parameter estimation}

The shape parameter $\left(n_{s}\right)$ was estimated by the relationship given by Bhunya et al. (2003) as:

$$
\begin{array}{cc}
n_{s}=5.53 \beta_{s}^{1.75}+1.04 & \text { for } 0.01<\beta_{s}<0.35 \\
n_{s}=6.29 \beta_{s}^{1.998}+1.157 & \text { for } \beta_{s} \geq 0.35
\end{array}
$$

where $\beta_{\mathrm{s}}$ is a non dimensional parameter defined as the product of peak sediment flow rate $\left(\mathrm{q}_{\mathrm{ps}}\right)$ [Tons/hr/Tons] and time to peak sediment flow rate $\left(\mathrm{t}_{\mathrm{ps}}\right)$ [hr]. The rest of the parameters were estimated by using the non-linear Marquardt algorithm (Marquardt, 1963) 
of the least squares procedure. In the present application, potential maximum erosion $\mathrm{A}$ is also taken as a parameter due to lack of their observations. The estimated parameters along with storm event values are given in Table 1 and 2.

\begin{tabular}{|c|c|c|c|c|c|}
\hline $\begin{array}{c}\mathrm{t}_{\mathrm{ps}} \\
\text { Date of Event }\end{array}$ & $\begin{array}{c}\mathrm{q}_{\mathrm{s}} \\
\text { (Tons/hr/Tons) }\end{array}$ & $\begin{array}{c}\beta_{\mathrm{s}} \\
(\mathrm{hr})\end{array}$ & $\begin{array}{c}\mathrm{Q}_{\mathrm{s}(\mathrm{o})} \\
(\text { Tons })\end{array}$ & $\begin{array}{c}\mathrm{Q}_{\mathrm{ps}(\mathrm{o})} \\
(\text { Tons } / \mathrm{hr})\end{array}$ \\
\hline July 17, 1983 & 0.38 & 2 & 0.76 & 2739 & 1025 \\
\hline August 21/22, 1983 & 0.418 & 2 & 0.836 & 2070 & 875 \\
\hline July 15, 1984 & 0.397 & 2 & 0.794 & 3145 & 1043 \\
\hline August 18/19, 1984 & 0.404 & 2 & 0.81 & 2105 & 743 \\
\hline September 1/2,1984 & 0.39 & 2 & 0.78 & 1205 & 475 \\
\hline September 17/18, 1984 & 0.41 & 2 & 0.82 & 963 & 392 \\
\hline
\end{tabular}

Table 1. Characteristics of storm events

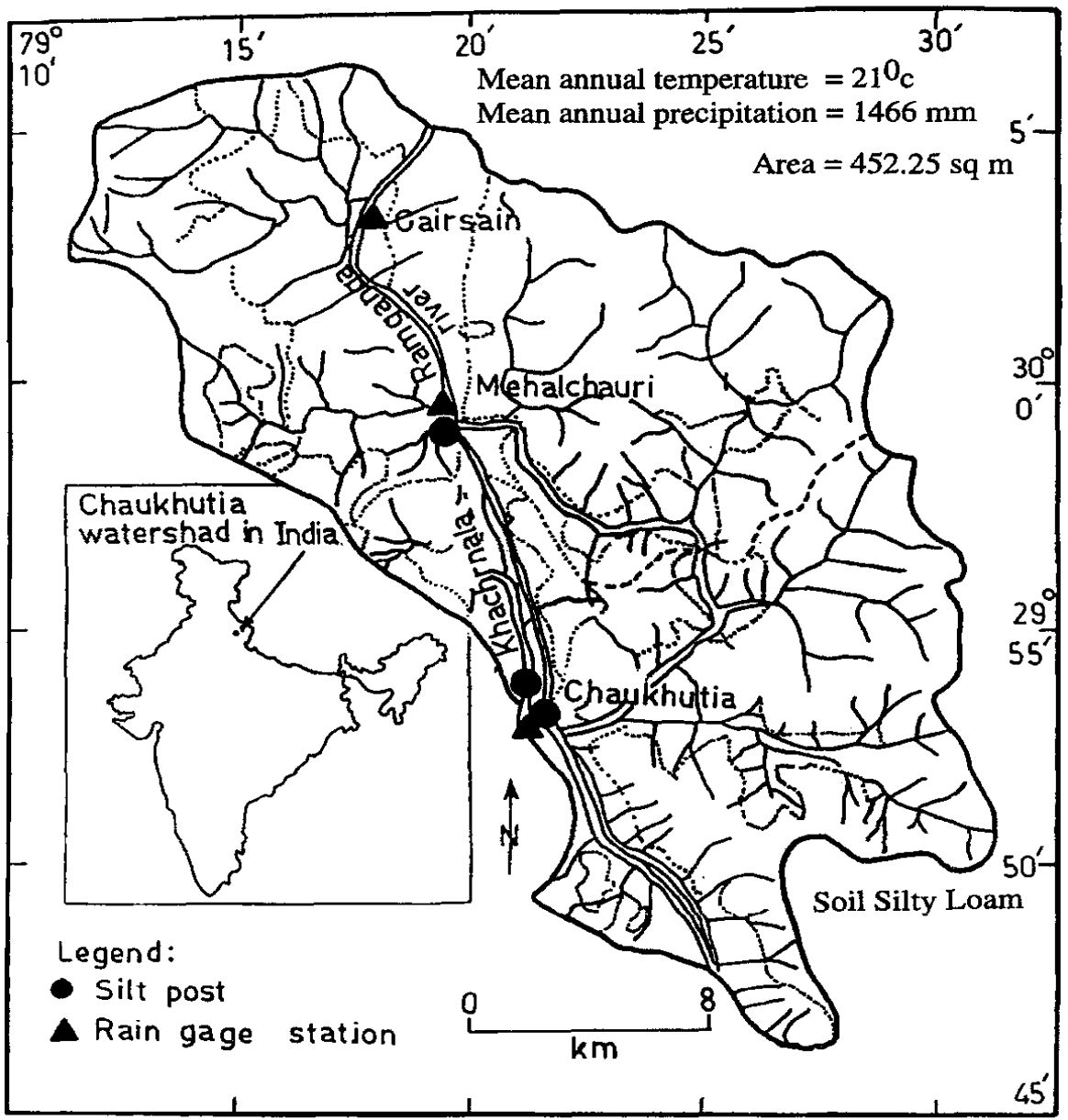

Fig. 18. Location of Chaukhutia watershed in Ramganga reservoir catchment (Source: Raghuwanshi et al. 1994) 


\begin{tabular}{|l|c|c|c|c|c|}
\hline Date of Event & \multicolumn{5}{|c|}{ Model parameters } \\
\hline & $\mathrm{n}_{\mathrm{s}}$ & $\mathrm{a}$ & $\beta$ & $\mathrm{k}$ & $\mathrm{A}\left(\right.$ Tons $\left./ \mathrm{Km}^{2}\right)$ \\
\hline July 17, 1983 & 4.79 & 0.530 & 0.351 & 0.029 & 26.66 \\
\hline August 21/22, 1983 & 5.55 & 0.727 & 0.701 & 0.030 & 40.78 \\
\hline July 15, 1984 & 5.12 & 0.735 & 0.721 & 0.030 & 62.69 \\
\hline August 18/19,1984 & 5.27 & 0.714 & 0.663 & 0.030 & 38.14 \\
\hline September 1/2,1984 & 4.99 & 0.388 & 0.425 & 0.030 & 19.64 \\
\hline September 17/18, 1984 & 5.39 & 0.587 & 0.781 & 0.030 & 29.34 \\
\hline
\end{tabular}

Table 2. Optimized parameter values for Chaukhutia watershed

\subsection{Performance of the proposed model}

The performance of the proposed sediment graph model was evaluated on the basis of their (i) closeness of the observed and computed sediment graphs visually; and (ii) goodness of fit (GOF) in terms of model efficiency (ME) and relative error (RE) of the results defined as:

$$
M E=1-\frac{\sum\left(Q_{s o}-Q_{s c}\right)^{2}}{\sum\left(Q_{s o}-\overline{Q_{s o}}\right)^{2}} ; R E(Q s)=\frac{Q_{s(o)}-Q_{s(c)}}{Q_{s(o)}} \times 100 ; R E(Q p s)=\frac{Q_{p s(o)}-Q_{p s(c)}}{Q_{p s(o)}} \times 100
$$

where $Q_{s(o)}$ and $Q_{s(c)}$ are observed and computed total sediment outflow, respectively $R E_{\left(Q_{s}\right)}$ and $\mathrm{RE}_{(\mathrm{Qps})}$ are relative errors in total sediment outflow and peak sediment flow rates, respectively.

For visual appraisal, the sediment graph computed using the proposed model is compared with the observed values using the data of August 18-19, 1984 event (Fig. 19). From the figure, it is observed that the computed sediment graph exhibits fair agreement with the observed graph. Similar results were also obtained for rest of the storm events that are not reported here. However, Fig. 20 \& 21 shows the comparison between computed and observed total sediment outflow and peak sediment outflow rates for all the storm events. The closeness of data points in terms of a best fit line and a value of $\mathrm{r}^{2} \approx 1.000$ indicate a satisfactory model performance for the assigned Job.

Further the results of GOF criteria given by Eq. (51) for all the events are shown in Table 3. The results indicate that the RE for total sediment outflow and peak sediment flow rate estimates vary from 2.49 to $10.04 \%$ and 12.59 to $16.56 \%$, respectively. Though error in case of peak sediment flow rate estimation is on higher side, this may be taken safely because even the more elaborate process-based soil erosion models are found to produce results with still larger errors (Vanoni 1975; Foster 1982; Hadley et al. 1985; Wu et al. 1993; Wicks and Bathurst 1996; Jain et al. 2005). Table 3 also shows the GOF in terms of ME for the storm events considered in the application. It is observed that ME varies from 90.52 to $95.41 \%$, indicating a satisfactory performance of the model for sediment graph computations. 


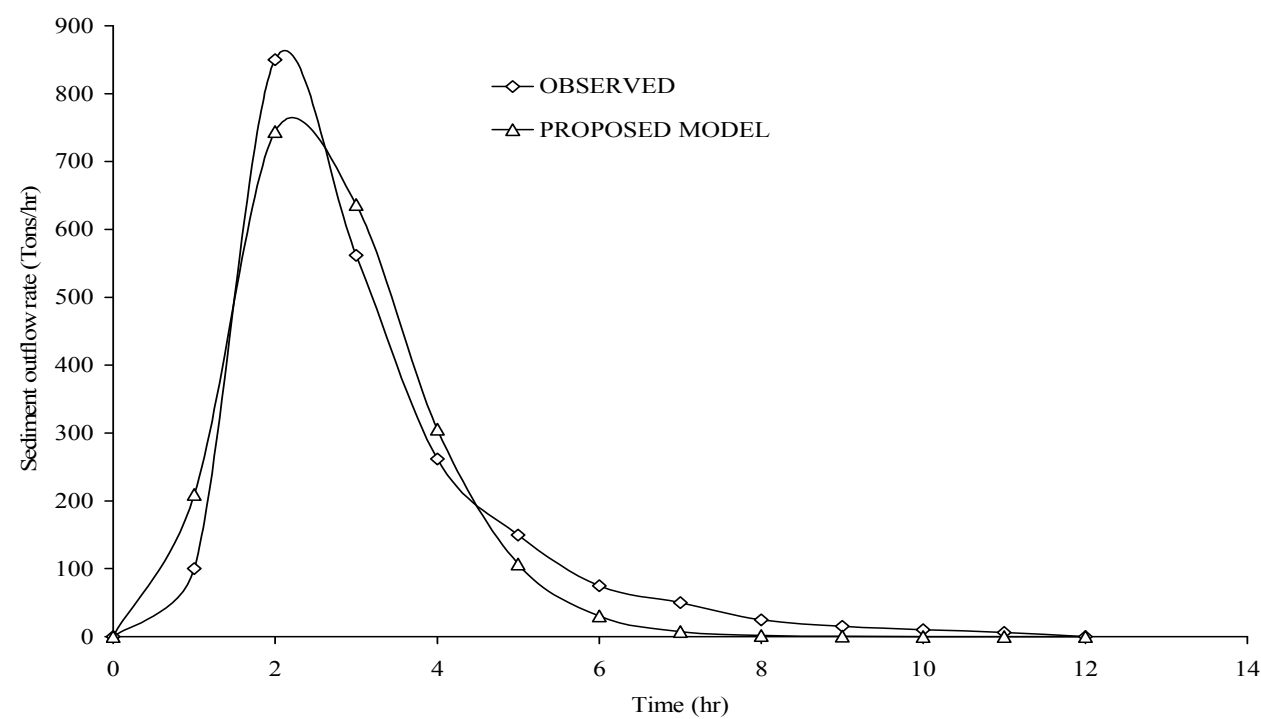

Fig. 19. Comparison of observed and computed sediment graphs for the storm of August, 18-19, 1984.

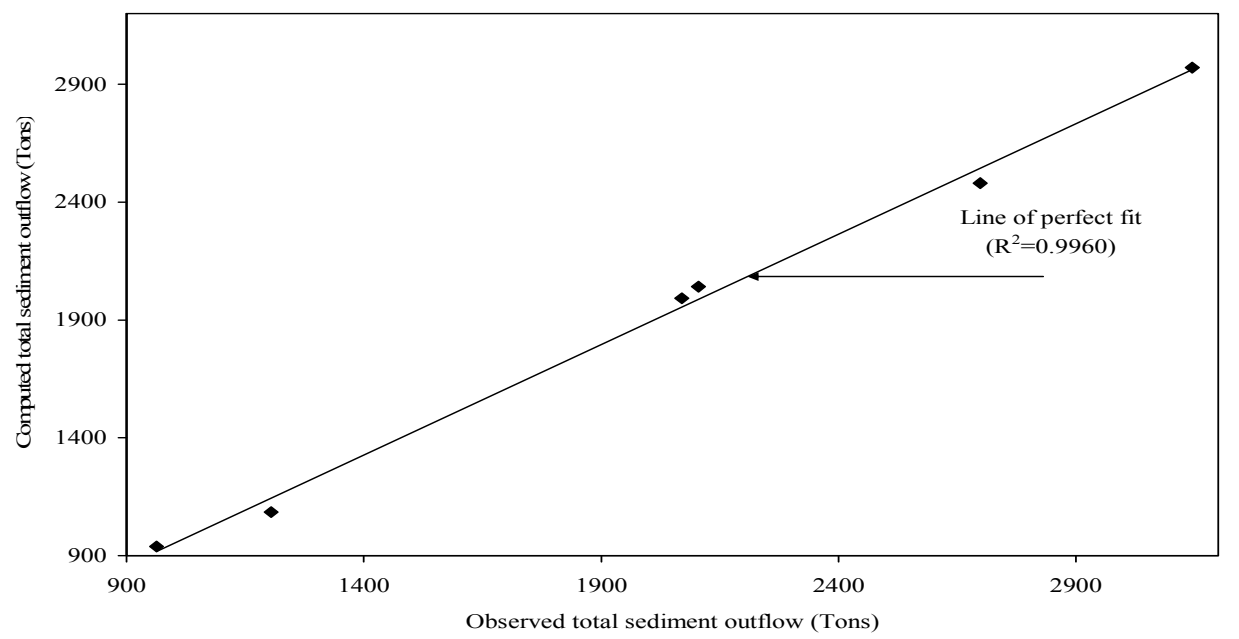

Fig. 20. Comparison between observed and computed total sediment outflow using proposed model for all storm events 


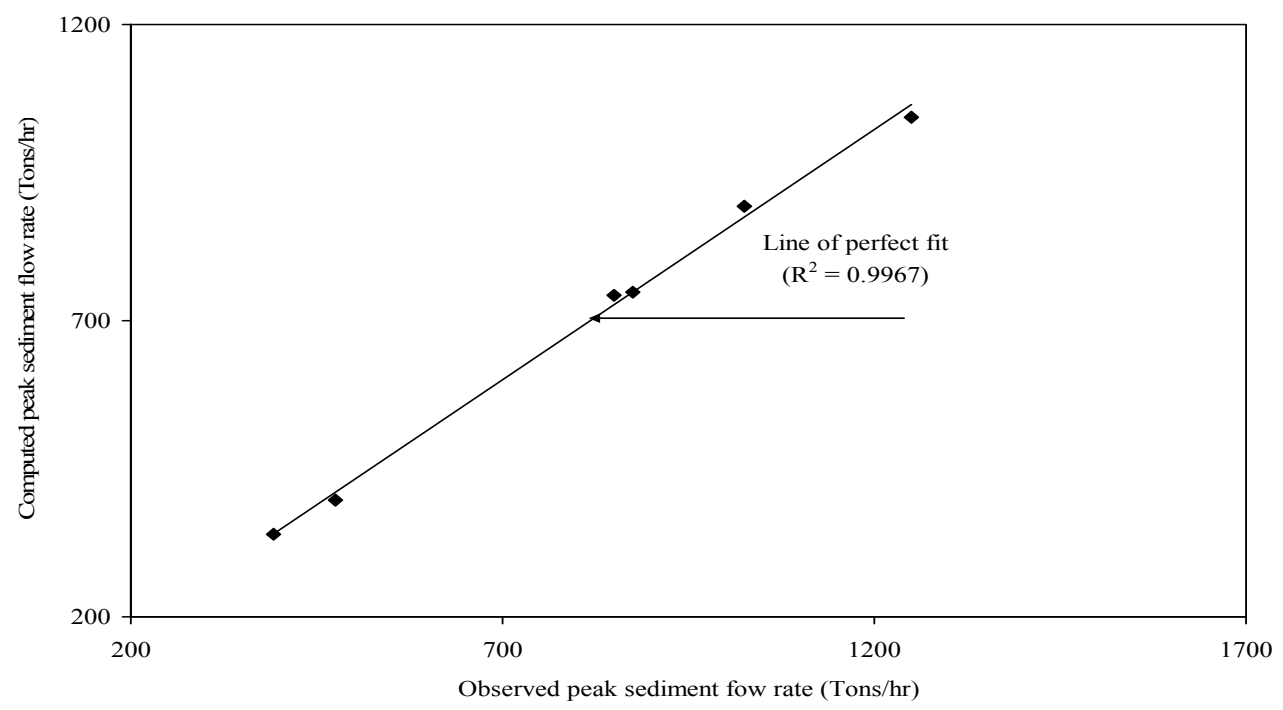

Fig. 21. Comparison between observed and computed peak sediment flow rates using proposed model for all storm events

\begin{tabular}{|l|c|c|c|}
\hline Date of Event & $\mathrm{RE}_{(\mathrm{QS})}$ & $\mathrm{RE}_{(\mathrm{Qps})}$ & Efficiency \\
\hline July 17, 1983 & 8.04 & 12.88 & 92.91 \\
\hline August 21/22, 1983 & 3.77 & 14.51 & 93.48 \\
\hline July 15, 1984 & 5.56 & 16.56 & 90.52 \\
\hline August 18/19, 1984 & 3.04 & 12.59 & 95.34 \\
\hline September 1/2,1984 & 10.04 & 16.42 & 93.65 \\
\hline September 17/18, 1984 & 2.49 & 13.52 & 95.41 \\
\hline
\end{tabular}

Table 3. Goodness of fit Statistics

\subsection{Sensitivity analysis}

From the results so far, it is imperative to analyze the sensitivity of different parameters of the proposed model for their effect on overall output. Here, the conventional analysis for sensitivity similar to the work of McCuen and Snyder (1986) and Mishra and Singh (2003) is followed as discussed in the following section.

It is evident form Eq. (49) that is a function of $\alpha, \beta, \mathrm{k}, \mathrm{n}$ and $\mathrm{A}$ i.e. $\mathrm{Q}_{\mathrm{s}}(\mathrm{t})=\mathrm{f}(\alpha, \beta, \mathrm{k}, \mathrm{n}, \mathrm{A})$. Therefore, the total derivative of $\mathrm{C}$ can be given as

$$
d Q_{s}(t)=\frac{\partial Q_{s}(t)}{\partial \alpha} d \alpha+\frac{\partial Q_{s}(t)}{\partial \beta} d \beta+\frac{\partial Q_{s}(t)}{\partial k} d k+\frac{\partial Q_{s}(t)}{\partial n} d n
$$

where $\frac{\partial Q_{s}(t)}{\partial \alpha}, \frac{\partial Q_{s}(t)}{\partial \beta}, \frac{\partial Q_{s}(t)}{\partial k}$ and $\frac{\partial Q_{s}(t)}{\partial n}$ are the partial derivatives of $Q_{s}(t)$ with respect to $\alpha, \beta, \mathrm{k}, \mathrm{n}$ respectively. The total derivative, $\mathrm{dQ}_{\mathrm{s}}(\mathrm{t})$, corresponding to the increments $\mathrm{da}$, 
$\mathrm{d} \beta, \mathrm{dk}$ and $\mathrm{dn}$ can be physically interpreted as the total variation of $\mathrm{Q}_{\mathrm{s}}(\mathrm{t})$ due to the variation of $\alpha, \beta, \mathrm{k}$ and $\mathrm{n}$ at any point in the $(\alpha, \beta, \mathrm{k}, \mathrm{n})$ domain. The variation of $\mathrm{Q}_{\mathrm{s}}(\mathrm{t})$ with respect to the variable under consideration can be derived from Eq. (49).

A more useful form of Eq. (52) can be given as

$$
\frac{d Q_{s}(t)}{Q_{s}(t)}=\left(\frac{\partial Q_{s}(t)}{\partial \alpha} \frac{\alpha}{Q_{s}(t)}\right) \frac{d \alpha}{\alpha}+\left(\frac{\partial Q_{s}(t)}{\partial \beta} \frac{\beta}{Q_{s}(t)}\right) \frac{d \beta}{\beta}+\left(\frac{\partial Q_{s}(t)}{\partial k} \frac{k}{Q_{s}(t)}\right) \frac{d k}{k}+\left(\frac{\partial Q_{s}(t)}{\partial n} \frac{n}{Q_{s}(t)}\right) \frac{d n}{n}
$$

where $\left(\frac{\partial Q_{s}(t)}{\partial \alpha} \frac{\alpha}{Q_{s}(t)}\right),\left(\frac{\partial Q_{s}(t)}{\partial \beta} \frac{\beta}{Q_{s}(t)}\right),\left(\frac{\partial Q_{s}(t)}{\partial k} \frac{k}{Q_{s}(t)}\right)$ and $\left(\frac{\partial Q_{s}(t)}{\partial n} \frac{n}{Q_{s}(t)}\right)$ are referred to as the ratio of the error in the sediment flow rate $\left(d Q_{s}(t) / Q_{s}(t)\right)$ to the error in $a(d a / \alpha)$, to the error in $\beta(d \beta / \beta)$, to the error in $k(d k / k)$, and to the error in $n(d n / n)$. Now, individual ratio terms corresponding to each parameter can be derived from Eq. (49) as follows:

$$
\frac{\partial Q_{s}(t)}{\partial \alpha} \frac{\alpha}{Q_{s}(t)}=1
$$

A similar error ratio term for parameter 'A' $\left(\frac{\partial Q_{s}(t)}{\partial A} \frac{A}{Q_{s}(t)}\right)$ can be obtained as well.

Similarly, for rest of the parameters, the error ratio terms are derived as

$$
\begin{gathered}
\frac{\partial Q_{s}(t)}{\partial \beta} \frac{\beta}{Q_{s}(t)}=\beta \ln \left(\frac{k t}{1+k t}\right) \\
\frac{\partial Q_{s}(t)}{\partial k} \frac{k}{Q_{s}(t)}=\frac{\beta}{t(1+k t)} \\
\frac{\partial Q_{s}(t)}{\partial n} \frac{n}{Q_{s}(t)}=\frac{[(n-1)(2-n) \ln c-(6-3.5 n] n}{(n-1)^{2.5}}, \text { where } \mathrm{c}=\left(t / t_{p}\right) \exp \left(-t / t_{p}\right), \mathrm{n}>1
\end{gathered}
$$

Eq. (57) is based on the expansion of exponential term up to first order only.

\section{Sensitivity to a}

In order to analyze the model sensitivity to parameter a the terms pertaining to $\beta, \mathrm{k}$ and $\mathrm{n}$ are eliminated from Eq. (53) and the resulting expression reduces to

$$
\frac{d Q_{s}(t)}{Q_{s}(t)}=\left(\frac{\partial Q_{s}(t)}{\partial \alpha} \frac{\alpha}{Q_{s}(t)}\right) \frac{d \alpha}{\alpha}
$$

Coupling of Eqs. (58) and (54) results

$$
\frac{d Q_{s}(t)}{Q_{s}(t)}=\frac{d \alpha}{\alpha}, \text { or } \frac{d Q s(t) / Q s(t)}{d \alpha / \alpha}=1
$$


From Eq. (59) it can be inferred that the ratio of the error in $Q_{s}(t)$ to the error in $a$ is 1 . This indicate that the any variation (increase or decrease) in a estimates will cause a same amount of variation (increase or decrease) in $\mathrm{Q}_{\mathrm{s}}(\mathrm{t})$, as depicted in Fig. 22. Similar pattern can be observed for parameter A also.

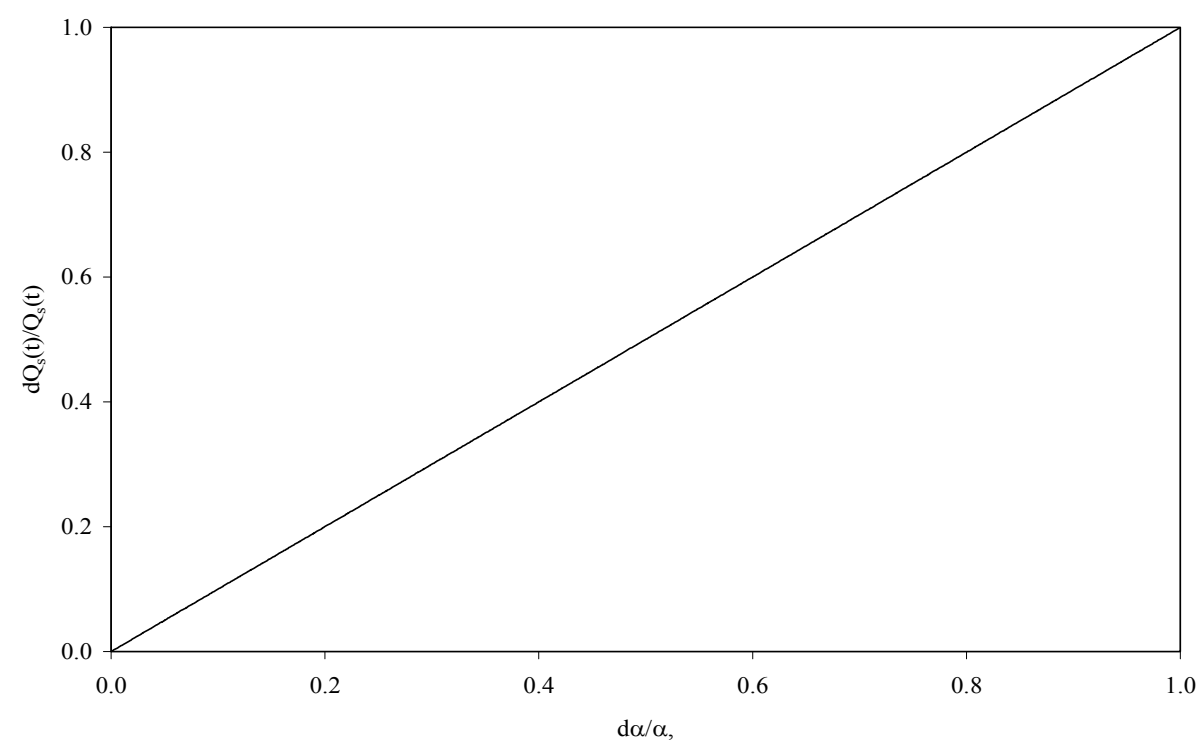

Fig. 22. Sensitivity of sediment outflow rate to a

\section{Sensitivity to $\beta$}

Similar to the above, the variation of $\beta$ only is considered after ignoring the impact of $\alpha, k$, and n, Eq. (38) in such case reduces to the following form

$$
\frac{d Q_{s}(t)}{Q_{s}(t)}=\left(\frac{\partial Q_{s}(t)}{\partial \beta} \frac{\beta}{Q_{s}(t)}\right) \frac{d \beta}{\beta}
$$

or

$$
\frac{d Q_{s}(t) / Q s(t)}{d \beta / \beta}=\left(\frac{\partial Q_{s}(t)}{\partial \beta} \frac{\beta}{Q_{s}(t)}\right)
$$

Equating Eqs. (61) and (55) one gets

$$
\frac{d Q_{s}(t) / Q s(t)}{d \beta / \beta}=\beta \ln \left(\frac{k t}{1+k t}\right)
$$

Analogous to the previous analysis, the left hand side of Eq. (62) represents the ratio of error in $Q_{s}(t)$ to the error in $\beta$, and the same is shown in Fig. 23 . It is apparent from Fig. 23 that any variation (increase) in $\beta$ for a given $t$ and $k$ causes $Q_{s}(t)$ to decrease. 


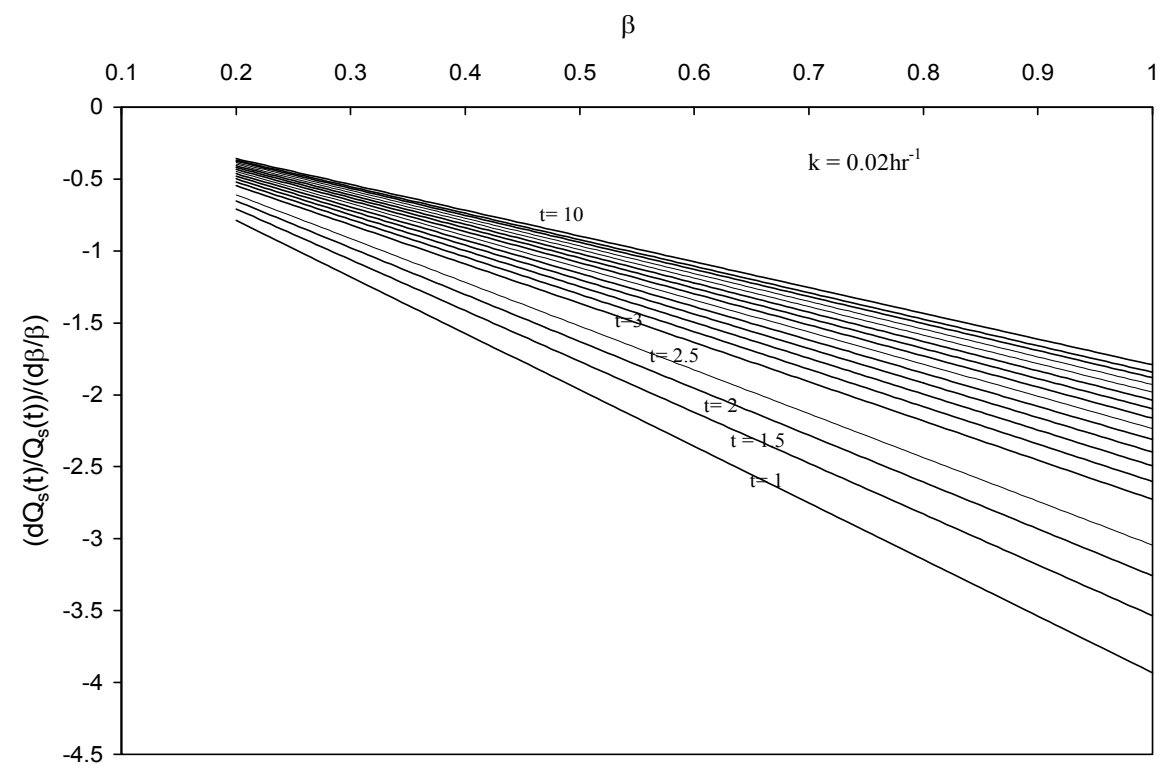

Fig. 23. Sensitivity of sediment outflow rate to $\beta$

\section{Sensitivity to $\mathrm{k}$}

As above, considering the variation of $k$ only reduces Eq. (53) reduces to the following form.

$$
\frac{d Q_{s}(t)}{Q_{s}(t)}=\left(\frac{\partial Q_{s}(t)}{\partial k} \frac{k}{Q_{s}(t)}\right) \frac{d k}{k}
$$

Alternatively, Eq. (48) can be expressed as

$$
\frac{d Q_{s}(t) / Q s(t)}{d k / k}=\left(\frac{\partial Q_{s}(t)}{\partial k} \frac{k}{Q_{s}(t)}\right)
$$

Equating Eqs. (64) and (56) one gets

$$
\frac{d Q_{s}(t) / Q s(t)}{d k / k}=\frac{\beta}{t(1+k t)}
$$

As expressed in Eq. (65) and shown in Fig. 24, for any increase in $\mathrm{k}$ the ratio of errors tends to decrease, implying the $Q_{s}(t)$ to increase and vice versa.

\section{Sensitivity to $\mathbf{n}$}

Similar to the preceding analysis, if the variation of only $n$ is considered ignoring the impact of $a, \beta, k$, Eq. (53) reduces to

$$
\frac{d Q_{s}(t)}{Q_{s}(t)}=\left(\frac{\partial Q_{s}(t)}{\partial n} \frac{n}{Q_{s}(t)}\right) \frac{d n}{n}
$$




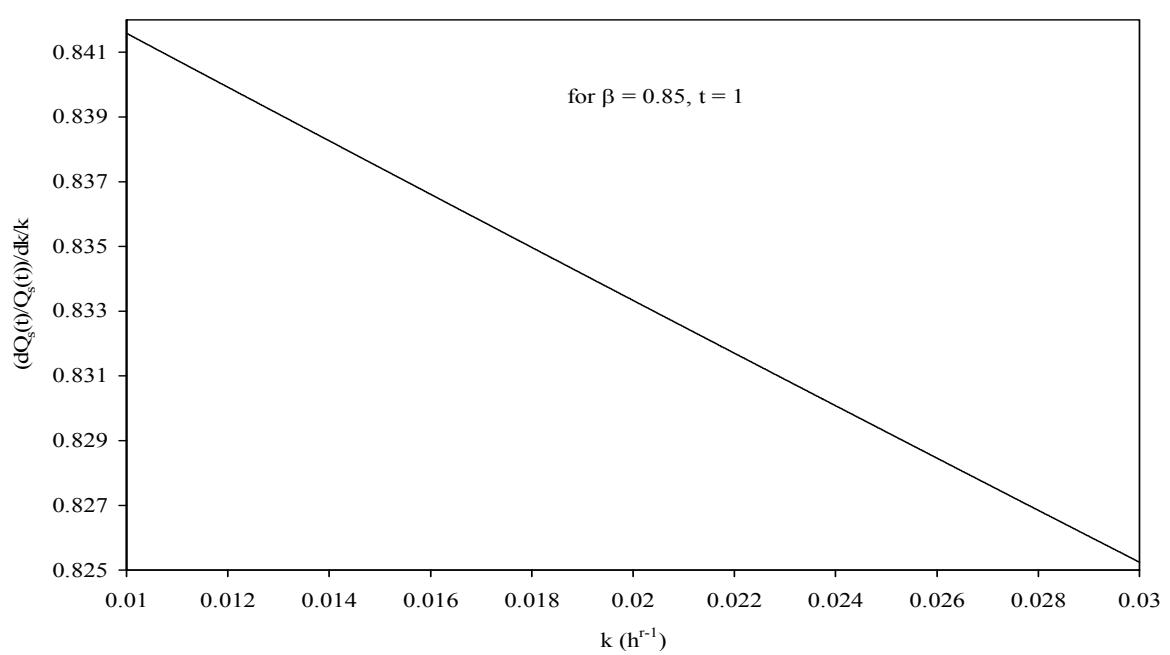

Fig. 24. Sensitivity of sediment outflow rate to $\mathrm{k}$

Equating Eqs. (66) \& (57) results

$$
\frac{d Q_{s}(t) / Q s(t)}{d n / n}=\frac{[(n-1)(2-n) \ln c-(6-3.5 n] n}{(n-1)^{2.5}}, \text { where } c=\left(t / t_{p}\right) \exp \left(-t / t_{p}\right), \mathrm{n}>1
$$

Analogous to the previous analysis, the left hand side of Eq. (67) represents the ratio of error in $Q_{s}(t)$ to the error in $n$. It is apparent from Fig. 25 that any variation (increase) in $n$ for a given $t / t_{p}$ causes the ratio to increase, implying $Q_{s}(t)$ to increase.

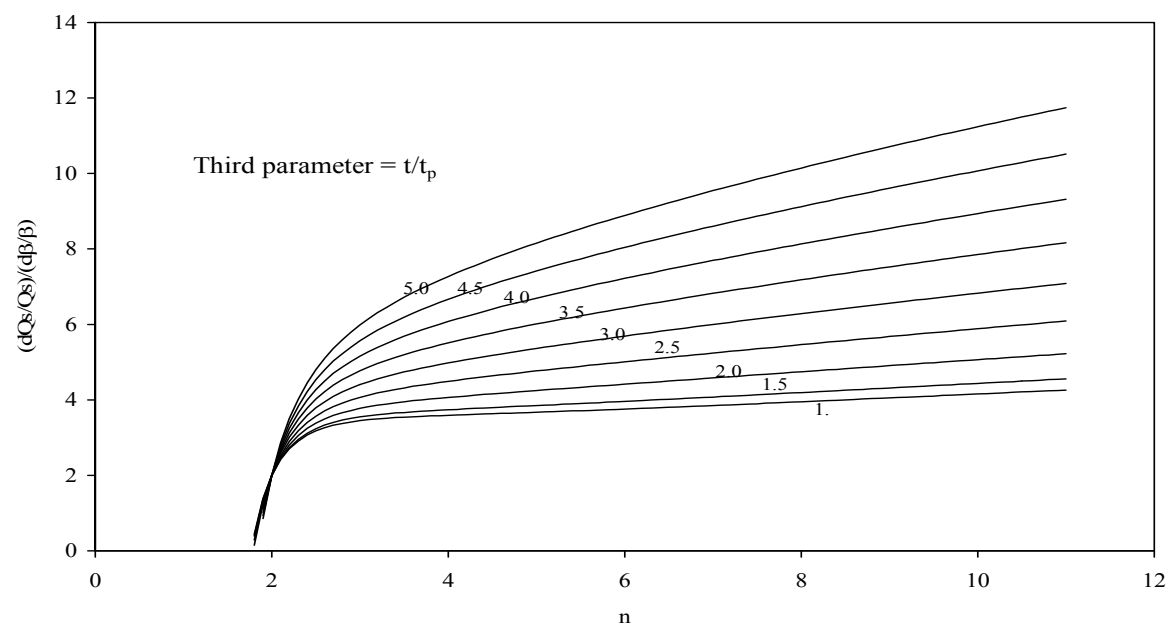

Fig. 25. Sensitivity of sediment outflow rate to $n$

A comparison of the sediment graphs computed by the proposed model and IUSG model (Raghuwanshi et al., 1994) with the observed sediment graph is shown in Fig. 26. It is 
observed that the proposed model compares more closely than IUSG with the observed sediment graph.

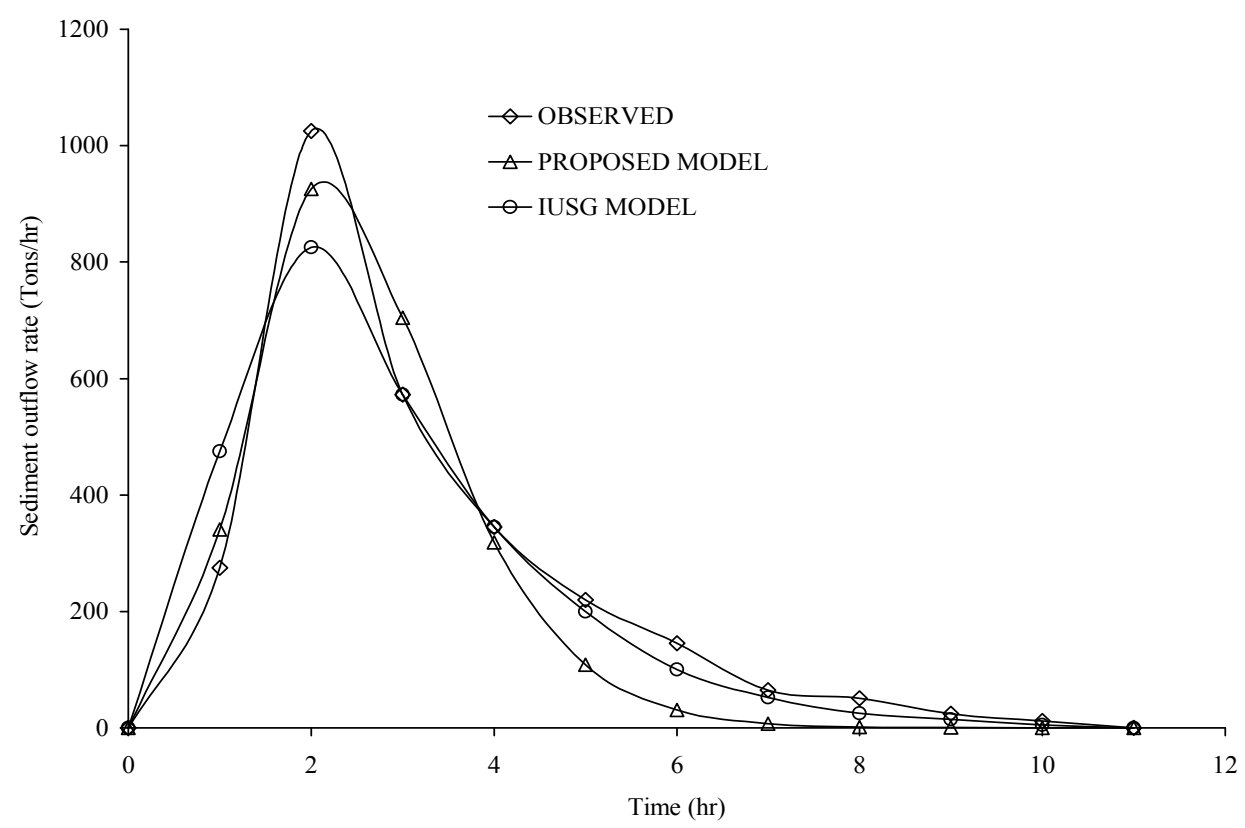

Fig. 26. Comparison of observed and computed sediment graphs for the storm of July 17, 1983.

\section{References}

Aksoy, H., and Kavvas, M. L., 2005. A review of hillslope and watershed scale erosion and sediment transport models. CATENA 64, 247-271.

Bhunya, P. K., Mishra, S. K., and Berndtsson, R., 2003. Simplified two-parameter gamma distribution for derivation of synthetic unit hydrograph. ASCE J. Hydrol. Eng. 8 (4), 226-230

Bogardi, I., Bardossy, A., Fogel, M., and Duckstein, L., 1986. Sediment yield from agricultural watersheds. ASCE J. Hydraul. Eng. 112 (1), 64-70.

Chen, V. J., and Kuo, C. Y., 1986. A study of synthetic sediment graphs for ungauged watersheds. J. Hydrol. 84, 35-54.

Chow, V. T., 1964. Applied Hydrology. McGraw-Hill, New York.

Foster, G. R., 1982. Modeling the erosion processes. In: C.T. Hann, Johnson, H., and Brakensiek, D.L. (Eds.), Hydrological modelling of small watersheds, ASAE Monograph No. 5, ASAE, St. Joseph, Mich.., 297-380.

Hadley, R. F., Lal, R., Onstad, C. A., Walling, D. E., and Yair, A., 1985. Recent developments in erosion and sediment yield studies. IHP-II Project A.1.3.1, UNESCO, Paris. 
Hawkins, R. H., Woodward, D. E., and Jiang, R., 2001. Investigation of the runoff curve number abstraction ratio. Paper presented at USDA-NRCS Hydraulic Engineering Workshop, Tucson, Arizona.

Horton, R. I., (1938) The interpretation and application of runoff plot experiments with reference to soil erosion problems. Proc. Soil Science Society of America, 3, 340-349.

Jain, M. K., Kothyari, U.C., and RangaRaju, K. G., 2005. GIS based distributed model for soil erosion and rate of sediment outflow from catchments. ASCE J. Hydraul. Eng. 131 (9), 755-769.

Kavvas, M. L., Chen, Z. Q., Dogrul, C., Yoon, J. Y., Ohara, N., Liang, L., Aksoy, H., Anderson, M. L., Yoshitani, J., Fukami, K., and Matsuura, T., 2004. Watershed environmental hydrology (WEHY) model based on upscaled conservation equations: hydrologic module. ASCE J. Hydrol. Eng. 9 (6), 450-464.

Kavvas, M. L., Yoon, J. Y., Chen, Z. Q., Liang, L., Dogrul, C., Ohara, N., Aksoy, H., Anderson, M. L., Reuters, J., and Hackley, S., 2006. Watershed environmental hydrology model: Environmental module and its application to a California watershed. ASCE J. Hydrol. Eng. 11 (3), 261-272.

Kothyari, U. C., Tiwari, A. K., and Singh, R., 1996. Temporal variation of sediment yield. ASCE J. Hydrol. Eng. 1 (4), 169-176.

Kumar, S., and Rastogi, R. A., 1987. Conceptual catchment model for estimation of suspended sediment flow. J. Hydrol. 95, 155-163.

Lee, Y. H., and Singh, V. P., 1999. Prediction of sediment yield by coupling Kalman filter with instantaneous unit sediment graph. Hydrol. Process. 13, 2861-2875.

Lee, Y. H., and Singh, V. P., 2005. Tank model for sediment yield. Water Resour. Mgmt. J. 19, 349-362.

Marquardt, D.W., 1963. An algorithm for least-square estimation of non-linear parameters. J. Soc. Indust. Appl. Math. 11 (2), 431-441.

McCuen, R.H., 2002. Approach to confidence interval estimation for curve numbers. ASCE J. Hydrol. Eng. 7 (1), 43-48.

Mein, R. G., and Larson, C. L., 1971. Modeling the infiltration component of the rainfallrunoff process. Water Resources Research Center, University of Minnesota, Graduate School, Minneapolis, Minnesota.

Merritt, W.S., Letcher, R.A., and Jakeman, A.J., 2003. A review of erosion and sediment transport models. Environ. Model. Software 18, 761-799.

Michel, C., Andreassian, V., and Perrin, C., 2005. Soil Conservation Service Curve Number method: how to mend a wrong soil moisture accounting procedure. Water Resour. Res. 41.

Mishra, S. K., and Singh, V. P., 1999. Another look at SCS-CN method. ASCE J. Hydrol. Eng. 4 (3), 257-264.

Mishra, S. K., and Singh, V. P., 2003. Soil Conservation Service Curve Number (SCS-CN) Methodology. Kluwer Academic Publishers, P.O. Box 17, 3300 AA, Dordrecht, The Netherlands.

Mishra, S. K., and Singh, V. P., 2004. Validity and extension of the SCS-CN method for computing infiltration and rainfall-excess rates. Hydrol. Process. 18 (17), 3323-3345.

Mishra, S. K., Tyagi, J.V., Singh, V. P., and Singh, R., 2006. SCS-CN based modeling of sediment yield. J. Hydrol. 324, 301-322. 
Nash, J. E., 1957. The form of instantaneous unit hydrograph. Bull. Int. Assoc. Sci. Hydrol. 3, 114-121.

Nash, J.E., and Sutcliffe, J.V., 1970 River flow forecasting through conceptual models. Part I A discussion of principles. J. Hydrol. 10, 282-290.

Novotny, V., and Olem, H., 1994. Water Quality: Prevention, Identification, and Management of Diffuse Pollution. John Wiley \& Sons, New York, N.Y.

Ponce, V.M., and Hawkins, R. H., 1996. Runoff curve number: has it reached maturity?. ASCE J. Hydrol. Eng. 1 (1), 11-19.

Raghuwanshi, N. S., Rastogi, R. A., and Kumar S., 1994. Instantaneous unit sediment graph. ASCE J. Hydraul. Eng. 120 (4) 495-503.

Rendon-Herrero, O., 1978. Unit sediment graph. Water Resour. Res. 14(5), 889-901.

SCS, 1956. National Engineering Handbook, Hydrology, Section 4, Soil Conservation Service, USDA, Washington DC.

Sharma, K. D., and Murthy, J. S. R., 1996. A conceptual sediment transport model for arid regions. J. Arid Environ. 33, 281-290.

Soil and Water Conservation Division (SWC\&D), 1991-1996. Evaluation of hydrological data, Vols. I and II, Ministry of Agriculture, Government of India, New Delhi, India.

Vanoni, V. A., ed. 1975. Sedimentation Engineering, ASCE, New York.

Wicks, J. M., and Bathurst, J. C. 1996. SHESED: A physically based, distributed erosion and sediment yield component for the SHE hydrological modeling system. J. Hydrol. $175,213-238$.

Williams, J. R., 1975. Sediment routing for agriculture watersheds. Water Resour. Bull. 11 (5), 965-974.

Williams, J. R., 1978. A sediment graph model based on an instantaneous unit sediment graph. Water Resour. Res. 14(4), 659-664.

Wilson, B. N., Barfield, B. J., Moore, I. D., and Warner, R. C., 1984. A hydrology and sedimentology watershed model. II: Sedimentology component." Transaction ASAE, 17, 1378-1384.

Wischmeier, W.H. and Smith, D.D., 1978. Predicting rainfall-erosion losses-A guide to conservation planning. Agricultural Handbook No. 537, Science and Education Administration, USDA, Washington DC.

Wu, T. H., Hall, J. A., and Bonta, J. V., 1993. Evaluation of runoff and erosion models. ASCE J. Irrig. and Drain. Eng. 119(4), 364-382.

Young, R. A., Onstad, C. A., Bosch, D.D., and Anderson, W.P., 1987. AGNPS: An agricultural non-point source pollution model: a large water analysis model, U. S. Dept. of Agriculture, Cons. Res. Report No.35, p.77. 


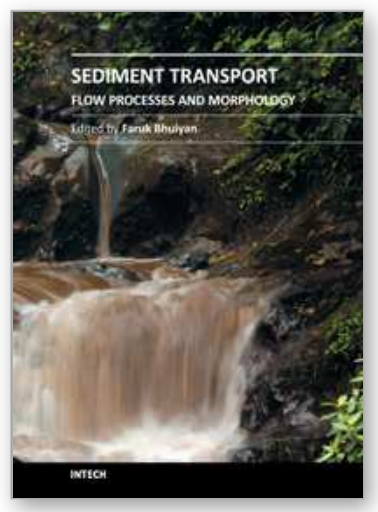

\section{Sediment Transport - Flow and Morphological Processes}

Edited by Prof. Faruk Bhuiyan

ISBN 978-953-307-374-3

Hard cover, 250 pages

Publisher InTech

Published online 26, October, 2011

Published in print edition October, 2011

The purpose of this book is to put together recent developments on sediment transport and morphological processes. There are twelve chapters in this book contributed by different authors who are currently involved in relevant research. First three chapters provide information on basic and advanced flow mechanisms including turbulence and movement of particles in water. Examples of computational procedures for sediment transport and morphological changes are given in the next five chapters. These include empirical predictions and numerical computations. Chapters nine and ten present some insights on environmental concerns with sediment transport. Last two contributions deal with two large-scale case studies related to changes in the transport and provenance of glacial marine sediments, and processes involving land slides.

\section{How to reference}

In order to correctly reference this scholarly work, feel free to copy and paste the following:

P. K. Bhunya, Ronny Berndtsson, Raj Deva Singh and S.N.Panda (2011). A Sediment Graph Model Based on SCS-CN Method, Sediment Transport - Flow and Morphological Processes, Prof. Faruk Bhuiyan (Ed.), ISBN: 978-953-307-374-3, InTech, Available from: http://www.intechopen.com/books/sediment-transport-flow-andmorphological-processes/a-sediment-graph-model-based-on-scs-cn-method

\section{INTECH}

open science | open minds

\section{InTech Europe}

University Campus STeP Ri

Slavka Krautzeka 83/A

51000 Rijeka, Croatia

Phone: +385 (51) 770447

Fax: +385 (51) 686166

www.intechopen.com

\section{InTech China}

Unit 405, Office Block, Hotel Equatorial Shanghai

No.65, Yan An Road (West), Shanghai, 200040, China

中国上海市延安西路65号上海国际贵都大饭店办公楼405单元

Phone: +86-21-62489820

Fax: +86-21-62489821 
(C) 2011 The Author(s). Licensee IntechOpen. This is an open access article distributed under the terms of the Creative Commons Attribution 3.0 License, which permits unrestricted use, distribution, and reproduction in any medium, provided the original work is properly cited. 